Images du travail, travail des images

\title{
Les usages « sauvages » de l'image. Retours sur une expérience profane de la sociologie visuelle
}

"Wild" uses of images. Experience feedbacks on an ordinary practice of visual sociology

Flora Bajard

\section{OpenEdition}

\section{Journals}

Édition électronique

URL : http://journals.openedition.org/itti/1065

DOI : 10.4000/itti. 1065

Éditeur

Université de Poitiers

\section{Référence électronique}

Flora Bajard, «Les usages «sauvages » de l'image. Retours sur une expérience profane de la sociologie visuelle », Images du travail, travail des images [En ligne], 3 | 2017, mis en ligne le 01 février 2017, consulté le 14 avril 2021. URL : http://journals.openedition.org/itti/1065 ; DOI : https://doi.org/ 10.4000/itti.1065

Ce document a été généré automatiquement le 14 avril 2021

Images du travail, travail des images 


\title{
Les usages « sauvages » de l'image. Retours sur une expérience profane de la sociologie visuelle
}

\author{
"Wild" uses of images. Experience feedbacks on an ordinary practice of visual \\ sociology
}

Flora Bajard

1 Les rapports qu'entretiennent les chercheurs avec l'image sont ambivalents, et peuvent être typologisés ainsi: certains en font un usage central, notamment les anthropologues et ethnologues, qui développent un rapport parfois banalisé et donc en partie impensé avec les opérations de recherche consistant à photographier sur leur terrain ${ }^{1}$. D'autres chercheurs proposent de nombreuses réflexions, en particulier sur un usage "maximaliste " de l'image consistant à en faire un "facilitateur " pour la recherche. Un exemple désormais classique est l'usage de l'image pendant le travail de terrain, notamment à travers la "photo-elicitation interview" (Collier, 1986) consistant à mobiliser ces documents dans la relation d'enquête afin de la stimuler (pour une mise en pratique plus récente de cette conception interactionnelle de la photographie, voir par exemple (Meyer, 2014). Une troisième catégorie de chercheurs pourrait être constituée par ceux qui ne font pas ou peu usage de l'image. Dans ce cas, on observe souvent un relatif détachement, voire relâchement, à l'égard des ces dernières, y compris pour expliquer pourquoi il n'en est pas fait usage. Alors que tout choix méthodologique implique généralement un travail de réflexivité dans les travaux universitaires, tout particulièrement en sciences sociales, inclure quelques illustrations dans un travail de thèse - ou ne pas le faire - semble ne pas devoir être davantage justifié. Ce constat est observable dans nombre de travaux et laboratoires, parfois désertés par les discussions autour de l'image. En fait, inclure de l'iconographie dans un article scientifique ou une thèse est souvent perçu comme un parti pris "sympathique » de la part d'un auteur souhaitant fournir quelques illustrations de son terrain et rendre plus agréable la lecture d'un travail. Ainsi, «les données visuelles récoltées (affiches, dépliants, flyers, journaux, film, photographies, etc.) n'apparaissent 
pas dans le produit final des recherches sociologiques, ou alors sous des formes d'annexes", conçues comme "de simples données de terrain non destinées à être restituées » (Chauvin et Reix, 2015, 17). La recherche doctorale qui fonde cet article est illustrative de ce cas de figure.

Une cause centrale de ce moindre intérêt provient notamment de la position des sciences sociales dans la hiérarchie scientifique: l'image viendrait apporter des éléments "trompeurs" dans une entreprise de recherche déjà soupçonnée d' " essayisme", par comparaison avec la supposée neutralité des sciences dites dures. Ceci explique sans doute la plus grande légitimité de l'écrit dans l'habitus scientifique en sciences sociales (Terrenoire, 1985). On peut aussi citer une dimension visuelle des objets peu explicitée ou peu perçue, ou encore la marginalité du corps humain dans la discipline (Piette, 2007, 24). Ces pistes sur les faibles usages de l'iconographie - ou ses usages en partie impensés et assez spontanés, renvoient à un niveau généraliste relevant finalement d'une autoanalyse des sciences sociales elles-mêmes. Dans cet article, c'est à ma pratique individuelle que je souhaite appliquer ces questionnements. S'il apparaît que cette démarche est souvent « justifiée par le sentiment que [la] récolte [de ces données] ne s'est pas faite selon une méthodologie assez rigoureuse » (Chauvin et Reix, 2015, 17), que recouvre plus précisément ce déficit méthodologique? Quels enseignements en tirer malgré tout sur le plan épistémologique, méthodologique et analytique?

3 Autrement dit, cet article vise à examiner ces usages parcellaires, silencieux, plus ou moins rationalisés et contrôlés, voire ces non-usages de l'image : ce sont ces différents aspects qui sont ici subsumés dans l'adjectif «sauvage $»^{2}$. C'est aussi en ce sens que je parle d'usages profanes de l'image - c'est-à-dire non explicitement inscrits dans une démarche de sociologie visuelle (entendue au sens large et dans sa pluralité : enquêter sur l'image ou avec l'image, ou encore faire de la sociologie en image (Maresca et Meyer, 2013)). Un retour réflexif sur ces usages met en évidence divers usages et potentiels, dont le repérage constitue un levier pour l'enquête : j'ai exploré ma propre pratique d'enquêtrice, des facettes inexploitées du terrain, ou encore des caractéristiques de mon objet. L'idée qui structure cet article est qu'expliciter le rapport avec notre objet et nos matériaux participe quoi qu'il en soit d'une démarche aspirant à la neutralité axiologique (conçue non pas comme absence de valeurs, mais comme vigilance à l'égard de leur imposition sur les objets étudiés (Weber, 1965, 15)). Dans cette perspective appliquée aux données visuelles, les malentendus et usages « ratés » de certaines photographies au cours de l'enquête et les " perturbations » ainsi engendrées ont par exemple été interrogées en révélant les a priori du chercheur et ses questionnements, révélant par contraste certaines représentations et pratiques endogènes (Papinot, 2007). En ce sens, l'image n'est pas fondamentalement différente, par nature, d'autres types de matériaux, et il est possible de l'appréhender « non plus comme une simple illustration mais comme un matériau premier [...] au même titre donc qu'une série statistique, un entretien ou une archive » (Dézé, 2013, 21). Autrement dit, parce que les images ne sont pas dénuées «d'impuretés » (Schwartz, 1993), elles peuvent tout autant être un support et un outil d'avancée dans la recherche; c'est d'ailleurs l'objet de plusieurs pans de la littérature existant aujourd'hui autour de l'image en sciences sociales ${ }^{3}$. Je m'inscris dans cette perspective, sans toutefois limiter la réflexion à l'analyse de la place de l'image dans la relation d'enquête. Cette contribution analyse plutôt la place des images dans le temps long, pour partie pendant 
la période de thèse et pour partie a posteriori, et au croisement de relations sociales impliquant plusieurs acteurs: le chercheur, les enquêtés et les récepteurs de ces travaux de recherche réflexion. Dès lors, cet article ne constitue pas une contribution supplémentaire aux méthodes d'analyse des images (sur ce point, voir par exemple Dézé, 2013) ou à la sémiologie des images. Cette « approche technicienne » - mettant au centre les questions esthétiques, épistémologiques, c'est-à-dire les manières de fabriquer les images - est, certes, tout à fait indispensable si l'on veut faire de celles-ci plus qu'une illustration des analyses et des observations de terrain (Maresca, 2007, 65). Plutôt centrée sur les usages de l'image, cette contribution restitue ainsi trois types de pratiques, depuis la plus spontanée jusqu'à la plus réflexive au moment de sa mise en œuvre : comme bien des chercheurs, je me suis employée à prendre des photos et à collecter des documents iconographiques lors de la phase d'enquête, de manière spontanée et allant de soi. Pourtant, cette pratique en partie impensée est susceptible de devenir un véritable outil ethnographique. Par la suite, j'ai analysé certaines images produites par les enquêtés eux-mêmes en tant que matériaux, pratiques sociales essentielles dans la gestion de leurs carrières individuelles et de la représentation du groupe professionnel ; l'usage escamoté de ces données empiriques révèle, en miroir, le potentiel de tels matériaux, pour l'étude des mondes du travail, en particulier. Enfin, la phase de finalisation de la recherche fut l'occasion d'incertitudes sur la sélection d'images plus ou moins "belles", et leur rapport à ma pratique du texte: mes dilemmes autour de l'esthétique furent à la fois témoin de mon manque de formation à la pratique de l'image, et source d'avancées en réflexivité.

\section{L'enquête sur un métier artistique indépendant : un usage profane de l'iconographie}

4 J'ai enquêté entre 2009 et 2014 sur les céramistes d'art en France : ces professionnels sont aujourd'hui environ 2000 en France ${ }^{4}$. Entre l'art et l'artisanat, ils s'adonnent à des pratiques variées : céramique utilitaire (pichets, assiette, tasses, plats...), pièces uniques et sculpturales. Ils exercent sous des statuts différents (artiste, artisan, profession libérale, auto-entrepreneur), et évoluent dans plusieurs types d'espaces (galeries, boutiques à domicile, marchés de potiers, salons de métiers d'art...).

Cette enquête a intégré l'étude des carrières, incluant le travail comme le hors-travail (le métier en tant que "style de vie »), mais visait aussi à saisir le sens des activités collectives (associatives, commerciales, institutionnelles). Elle a reposé principalement sur une immersion ethnographique dans les lieux de vie, de travail et de socialisation des céramistes d'art, et sur 62 entretiens semi-directifs, la plupart du temps individuels, auprès de responsables associatifs et institutionnels non céramistes, mais en très grande majorité avec des céramistes. Cette enquête a par ailleurs été jalonnée de discussions informelles, ainsi que d'observations: directes (ateliers, marchés, vernissages, évènements institutionnels, moments festifs, etc.) et participantes, puisque mes deux parents exercent ce métier (sur les implications de cette relation de familiarité et les usages de ces effets d'enquête, voir (Bajard, 2013)). Ce travail s'appuie enfin sur l'exploitation d'un questionnaire (218 répondants, soit environ $10 \%$ de la population) et de la documentation professionnelle (archives syndicales, compte-rendu associatifs, supports de communication, affiches, etc.). 
Concernant les images, j'ai eu plusieurs usages qualitatifs ${ }^{5}$ relativement « basiques » de celles-ci, au sens où ils étaient, pour partie, impensés. D'abord, les supports visuels n'ont pas été utilisés dans la relation d'enquête. Ensuite, il y a peu de photos (et, de manière générale, d'iconographie) dans le manuscrit de thèse, et celles-ci se trouvent essentiellement dans les annexes. Ces images ont été de plusieurs types: les miennes, prises au cours de l'enquête de terrain; des images prises dans l'écheveau des relations sociales, produites par les enquêtés: il s'agit surtout de photographies que les céramistes utilisent pour se présenter, eux et leur travail, sur des supports de communication (sites internet personnels et sites de galeries d'art principalement). Enfin, il faut préciser que j'ai eu l'occasion de rencontrer un photographe qui s'intéressait, à travers l'objectif de son appareil, au même objet que moi : le monde de la céramique d'art, ainsi que le mode de vie et le travail de ceux qui exercent ce métier ${ }^{6}$. Après avoir établi un contact avec lui, j'avais envisagé de mettre certaines réalisations de ce photographe dans le manuscrit de thèse, images que je jugeais très belles par ailleurs, et dont le contenu comme la forme me semblaient tout à fait adaptés à la restitution de ma pratique ethnographique; j'ai finalement renoncé à utiliser ses images, comme je l'explique à la fin de cet article.

\section{Deux usages classiques de la photographie : prise de note et illustration}

7 Prendre des photographies sur son terrain d'enquête ou utiliser des images pour accompagner la restitution de travaux fait partie des usages aujourd'hui banals de la recherche en sciences sociales. Pour autant, ceux-ci méritent d'être pensés dans ce qu'ils produisent et révèlent. À ce titre, j'expose ici plusieurs effets indirects de ces usages classiques, qui peuvent être intégrés dans la boite à outils de l'enquêteur.

\section{De la photographie-prise de note au travail d'inventaire : interroger le regard ethnographique sur le travail}

8 J'ai utilisé la photographie comme de nombreux enquêteurs le font au cours de la phase de terrain, c'est-à-dire comme « journal de terrain visuel » (Conord, 2007, 16). Cela me permettait de faciliter à la fois la mémorisation, essentiellement des lieux, puis la description ethnographique réalisée à partir de la prise de note. C'est donc d'une simple « image-outil» dans son acception minimaliste dont il est ici question (Mauss, 1967) 7 : je me munissais d'un appareil photo par précaution, parce que je préférais avoir trop d'outils - en plus d'un carnet de terrain et du dictaphone - que pas suffisamment. Sans le formaliser si précisément à l'époque, ma démarche visait à favoriser un « encombrement» de l'image par la capture de détails lors de la prise de la photo, pas encore sélectionnés par l'œil d'observatrice (Piette, 2007, 25). Soucieuse du détail, la restitution avec minutie des lieux, leurs ambiances, les objets qui les habitent, la manière dont se structurent les espaces, etc., me semblait suffisamment difficile à réaliser dans le cours des interactions - formelles ou non - avec les enquêtés pour qu'il s'avère utile de prendre appui sur quelques clichés pris ça et là de manière plus ou moins furtive. En effet, «l'esthétique photographique est susceptible de critiquer, sur le terrain du réalisme, les falsifications auxquelles peut conduire l'objectivité du naturalisme, lequel tend à résorber le caractère chaotique, irrégulier, hétérogène du 
social» (Laplantine, 2007, 55). De plus, à ces difficultés de la description ethnographique s'ajoutait celle d'activer sur le champ une "réflexivité réflexe" (Bourdieu, 1993a, 1391) pour travailler au mieux la relation d'enquête, et en particulier faire un usage stratégique des "perturbations " générées par ma présence (Bajard, 2013). Cependant, aussi basique que soit ce premier usage de la photographie, un retour réflexif sur ce procédé s'avère instructif sur ce que révèle le choix des scènes photographiées ou non, ou encore le cadrage de celles-ci : c'est ce que montre ces deux usages de l'image comme prise de note sur les outils d'abord, puis sur les lieux du travail.

9 Pendant l'enquête, j'ai pris soin de photographier l'ordinaire, à tous les sens du terme. Il s'agissait de ne pas photographier l'extraordinaire du travail lui-même, au sens de processus et de résultat : concrètement, j'ai veillé à ne pas prendre en photo que des grandes et imposantes pièces de céramique, ni trop de techniques spectaculaires ou photogéniques (par exemple les scènes impliquant le feu: la sortie de pièces incandescentes du four, les cuissons au feu de bois). De même, si je trouvais certains outils relativement esthétiques, attrayants par leur rareté ou leur matériau (un tour à pied à l'ancienne, certains outils en bois, de grands fours réalisés en briques et aux formes originales), j'ai corrigé très vite les dérives qu'aurait pu susciter cet attrait en m'efforçant de photographier des objets qui ne "m'intéressaient pas " dans leur symbolique ou leur esthétique (par exemple des étagères en fer, des planches en contreplaqué, des fours électriques). Ainsi, mon carnet de terrain photographique fut composé de photographies relativement hétérogènes et représentatives de la diversité des techniques professionnelles employées au sein de ce métier, et témoignent d'un regard attentif à la pluralité des manières de travailler.

Corpus 1. Photographies 1, 2, 3, 4, 5 et 6 : images regroupées sur une page des Annexes de la thèse illustrant la diversité d'un outil de travail (le four), pour partie constituée de photos issues du carnet de terrain visuel : 
Photographie 1.

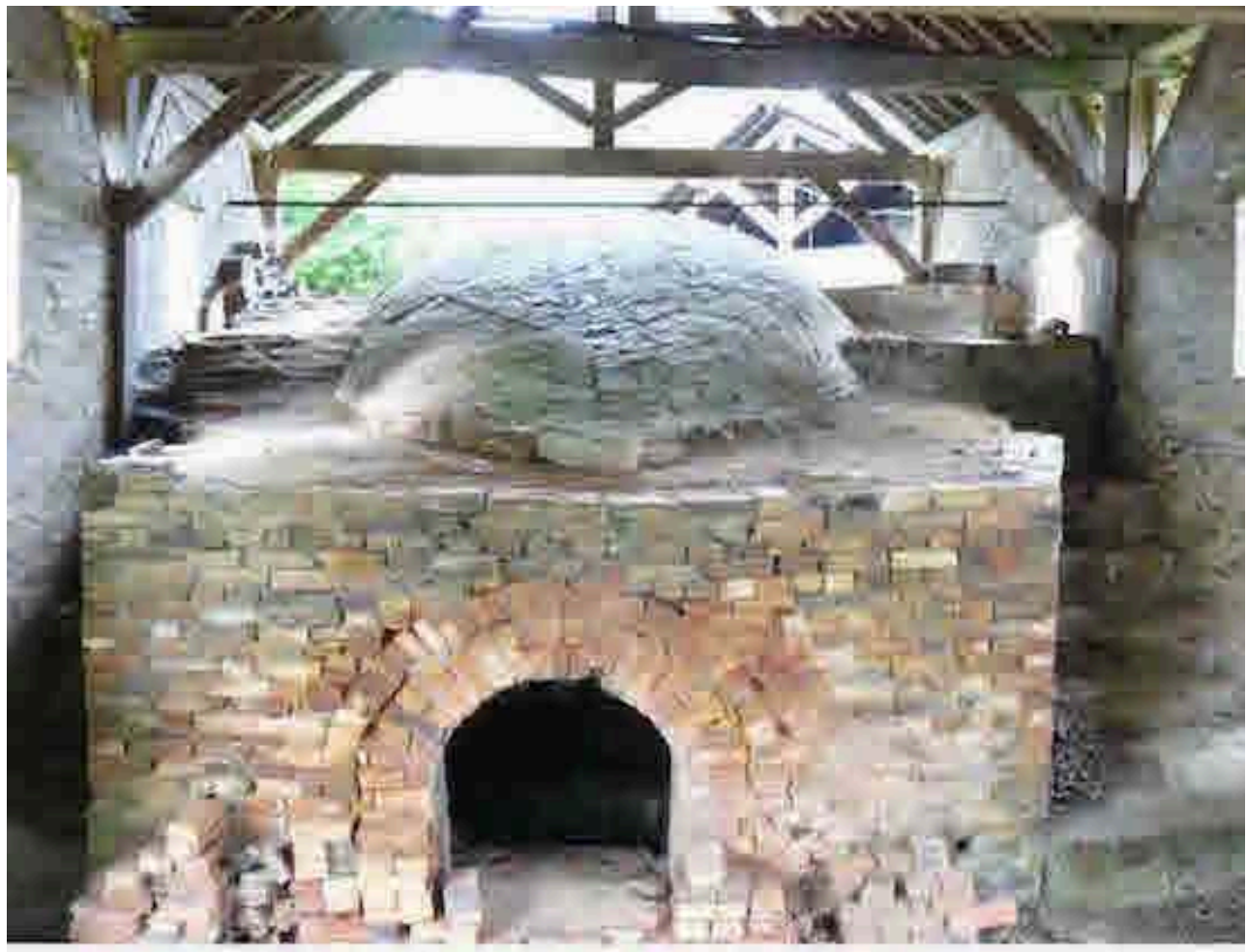

Le grand four à bois de La Borne, environ $70 \mathrm{~m}^{3}$.

10 La réduction de la taille des fours (photo 2. à bois et photo 3. électrique) qui illustre le passage d'une production utilitaire en grande série de singularité. 
Photographie 2.

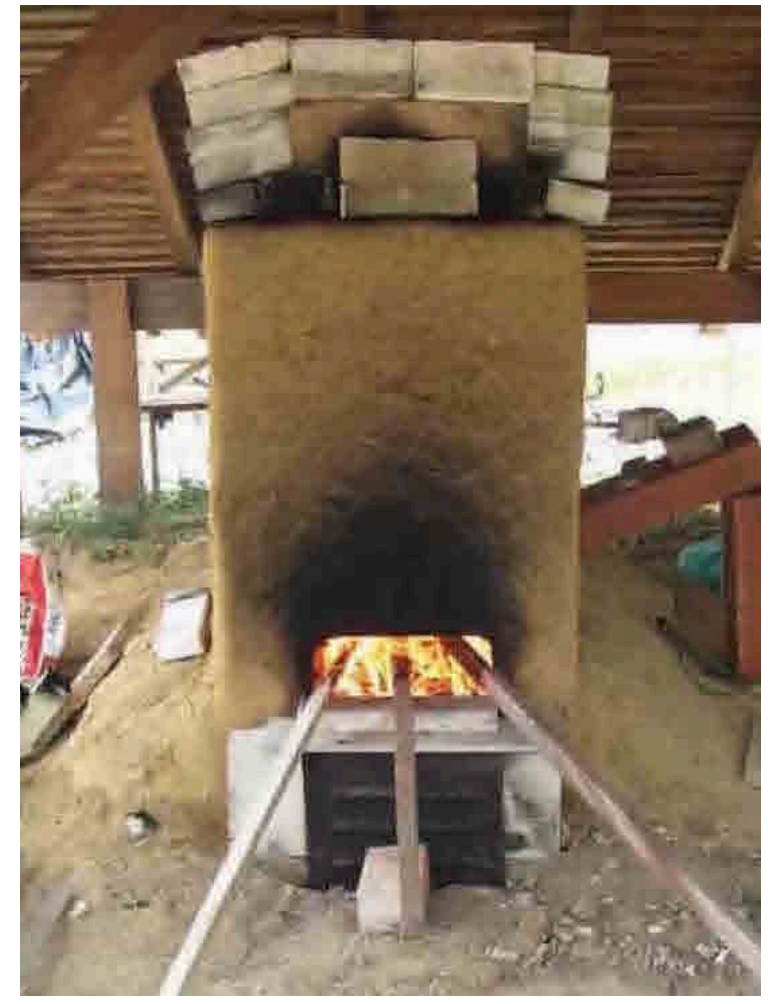

Four à bois.

Photographie 3.

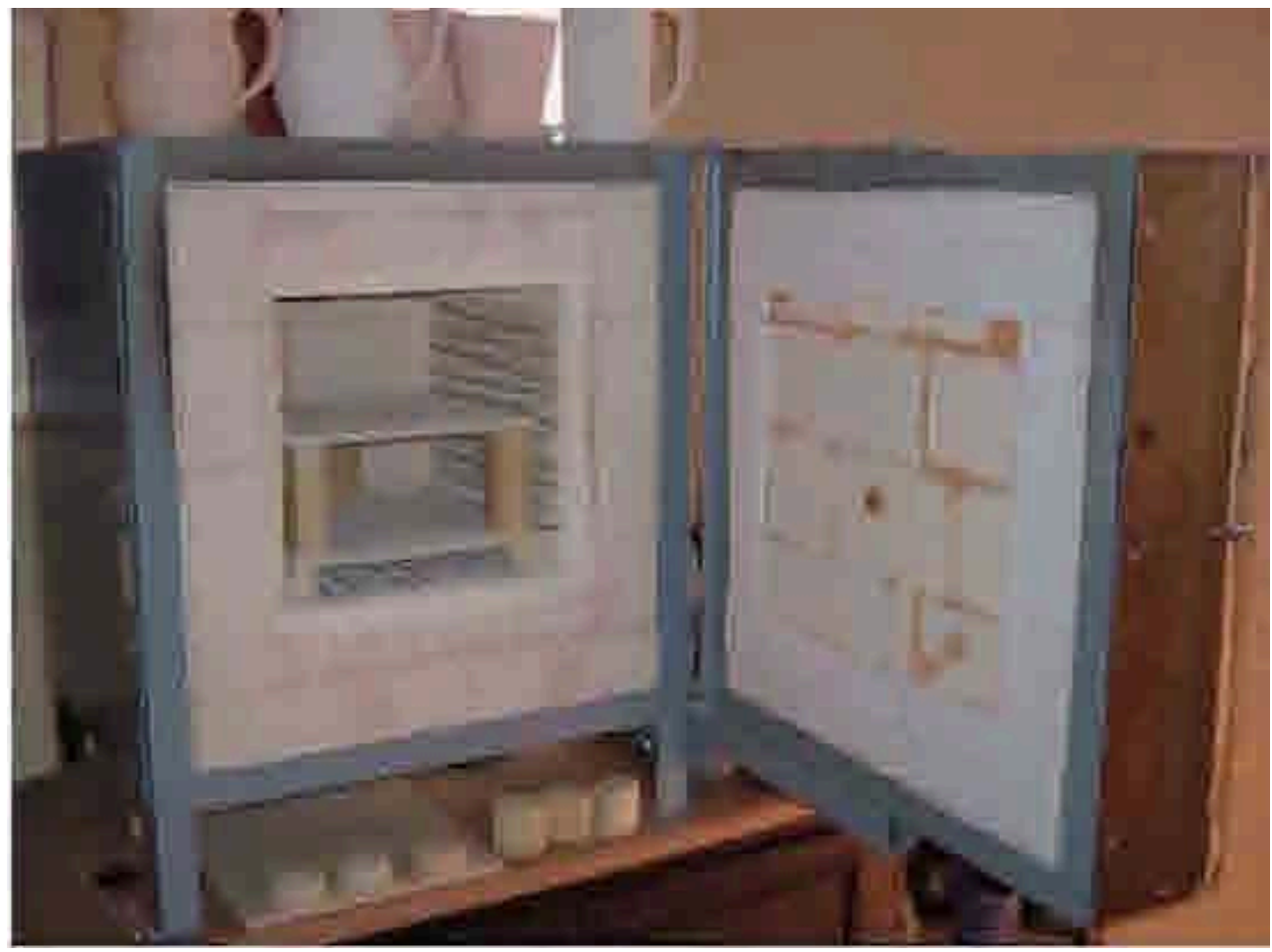

Four électrique. 
11 La multiplication des fours à bois dans le dernier quart du XXe siècle, plus ou moins originaux et personnalisés :

\section{Photographie 4.}

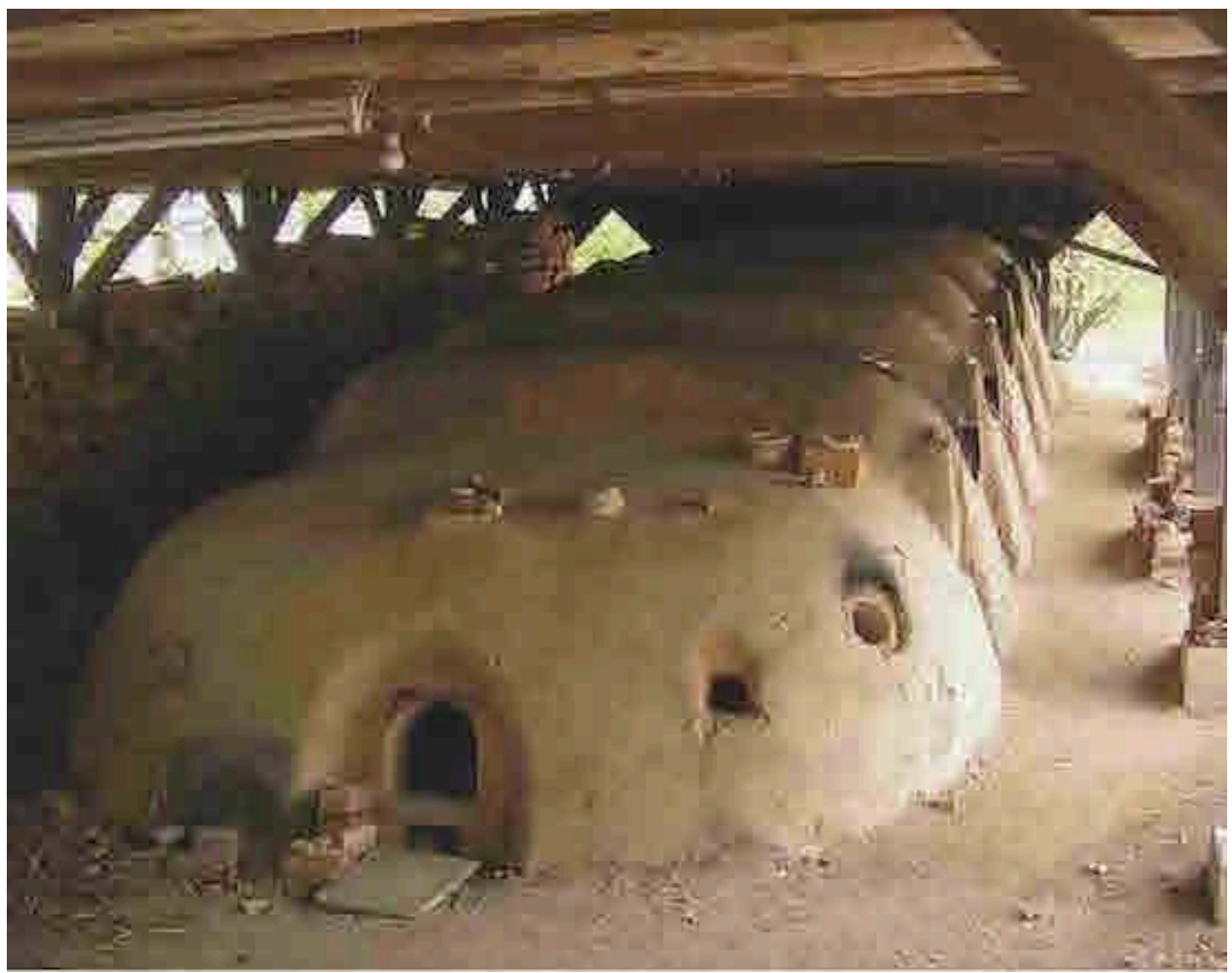

Four japonais « noborigama » 
Photographie 5.

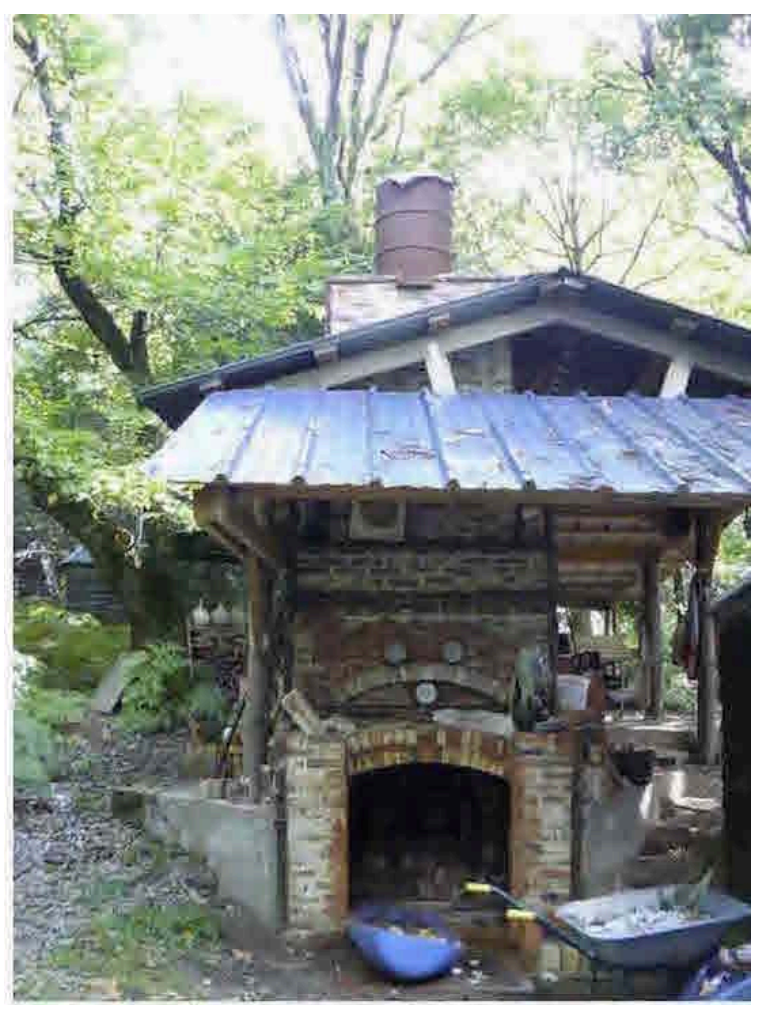

Four « escargot » inventé par son constructeur.

Photographie 6.

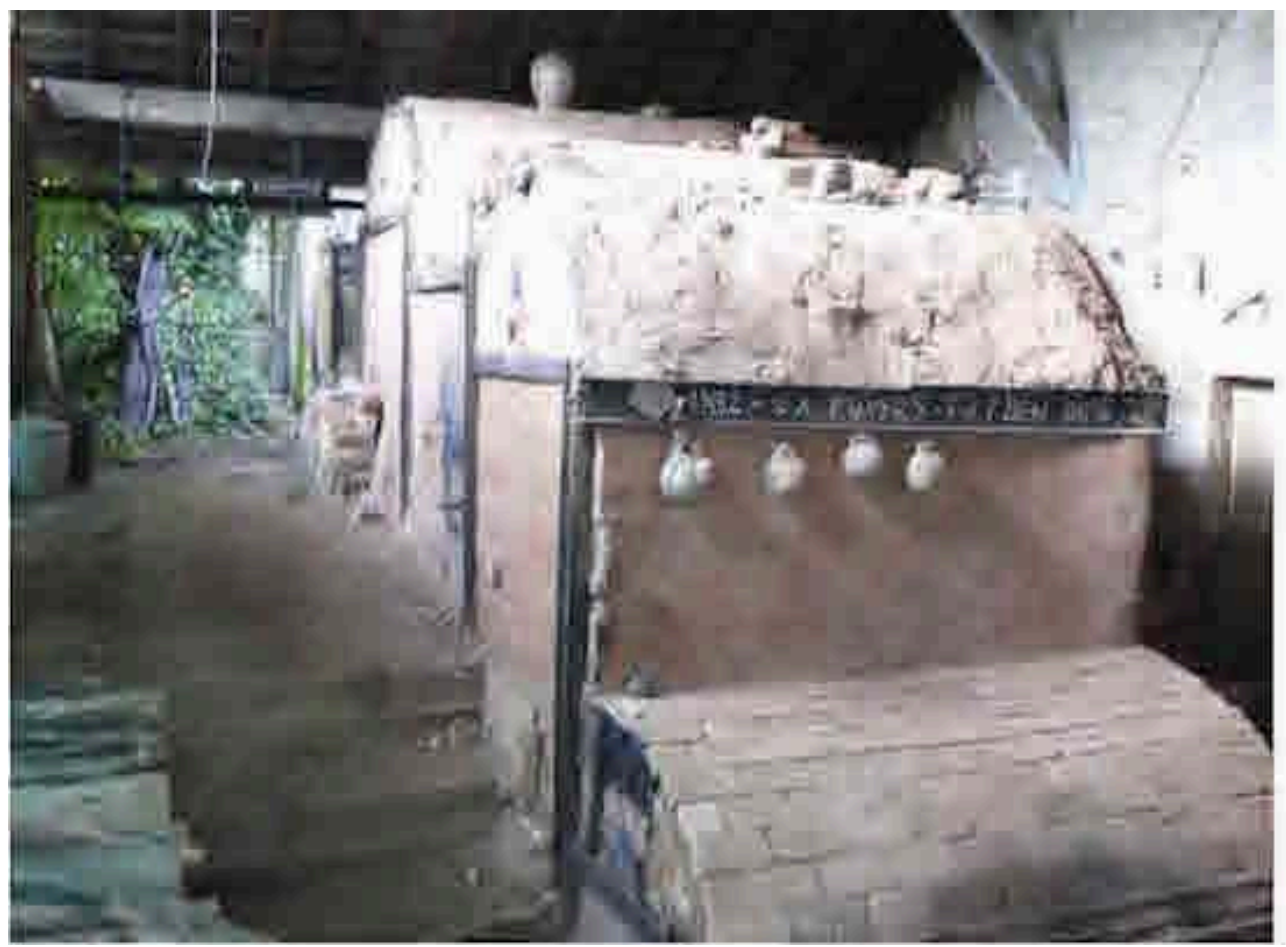

Four à trois chambres créé également par son constructeur. 
12 Pourtant, en appliquant ce raisonnement aux lieux du travail ou du hors-travail, c'est un autre travers qui est apparu. En effet, j'ai réalisé que j'avais des photos de l'intérieur des maisons des céramistes, car la notion de style de vie était déjà intégrée à ma grille d'analyse et je connaissais sa centralité dans les trajectoires des enquêtés. En revanche, je n'avais pas de photos des éventuels bureaux ou endroits où s'effectuent les tâches administratives (ce que les enquêtés appellent la "paperasse »), emblématiques du «sale boulot » (Hughes, 1996). Je n'ai en fait tout simplement pas pensé à demander à voir ces espaces. Certes, dans l'ethnographie, l'enquêteur est conduit à suivre de manière inductive le déroulé des événements; d'une certaine manière, il est donc logique que les céramistes ne m'aient pas présenté spontanément ces espaces de travail. Cependant, dans une conception active de la relation d'enquête conçue comme une relation sociale susceptible d'être construite et non dans une conception de l'ethnographe conçu comme simple "enregistreur » des faits et paroles indigènes, il n'aurait pas été inutile ni infondé de demander à voir ces endroits.

13 Ces deux exemples - l'un plutôt «réussi» (les outils de travail), l'autre plutôt " manqué » (les lieux) montrent qu'un usage en creux et a posteriori de la photographie pourrait être sa dimension de révélateur des regards posés sur l'objet. L'intérêt est que les scènes absentes ou présentes peuvent apparaître de manière sans doute plus évidente que ne peuvent le faire des descriptions ethnographiques textuelles. Ouvrir le stock d'images produites au cours de l'enquête et faire l'inventaire des lieux et des objets sur lesquels on s'est concentré, puis dresser, à l'inverse, la liste des lieux et objets dont on sait qu'ils existent mais qui restent introuvables, permet sans doute d'interroger les interstices du travail, ses zones d'ombres, informelles et peu gratifiantes, ou encore ses dimensions naturalisées et invisibilisées. L'invisibilisation de certains aspects du terrain peut procéder du chercheur ou des enquêtés, des habitudes issues de nos usages de la photographie dans la vie quotidienne, des contraintes du terrain enquêté. Qu'elles qu'en soient les raisons et dans une optique réflexive, ce sont alors finalement les métadonnées formées autour du corpus d'images qui nous intéressent. Dans un autre contexte, M. Meyer rappelle que deux types de commentaires peuvent en effet être produits à partir des images: les "données ethnographiques liées aux actions observées", et les "éléments rattachés aux moments de la prise de vue (Quand? Combien d'images? À quelle distance ? Quelles réactions ?) " (Meyer, 2014, 159). Si l'on ajoute à cette liste une question encore plus basique consistant à se demander ce qui a été photographié ou pas, nous pouvons faire de cette démarche d'inventaire un procédé supplémentaire à inclure dans la boite à outils de l'ethnographe.

\section{Confronter des profanes aux images, et produire un autre regard sur notre objet}

Un deuxième usage relativement commun que j'ai fait de l'image est l'utilisation d'illustrations, notamment face à des récepteurs de la recherche. Le seul statut d'illustration de l'image a largement été dépassé dans les travaux de sciences sociales, puisque l'image fait désormais partie intégrante des corpus de datas (sur ce basculement, voir (Pina et Savarese, à paraître)). Cependant, cet usage reste utile, puisqu'il "valorise la restitution des résultats de recherche et les clarifie parfois en apportant des éléments du monde visible qui aident à mieux faire une description » 
(Conord, 2007, 20). D'abord, je n'ai commencé à utiliser des images qu'après plusieurs mois de thèse, et ce, à travers deux supports. J'ai d'abord illustré certaines interventions orales (colloques, conférences...); je présentais alors une ou deux diapositives comportant des photographies.

Corpus 2. Images (photographies 7, 8, 9, 10, 11, 12, 13, 14 et 15) utilisées lors des premières restitutions de travaux (colloques, journées d'étude) : par cette sélection d'images produites par les enquêtés, j'ai produit une mise en scène renforçant la dimension « artifiée » des pièces représentées.

Photographie 7.

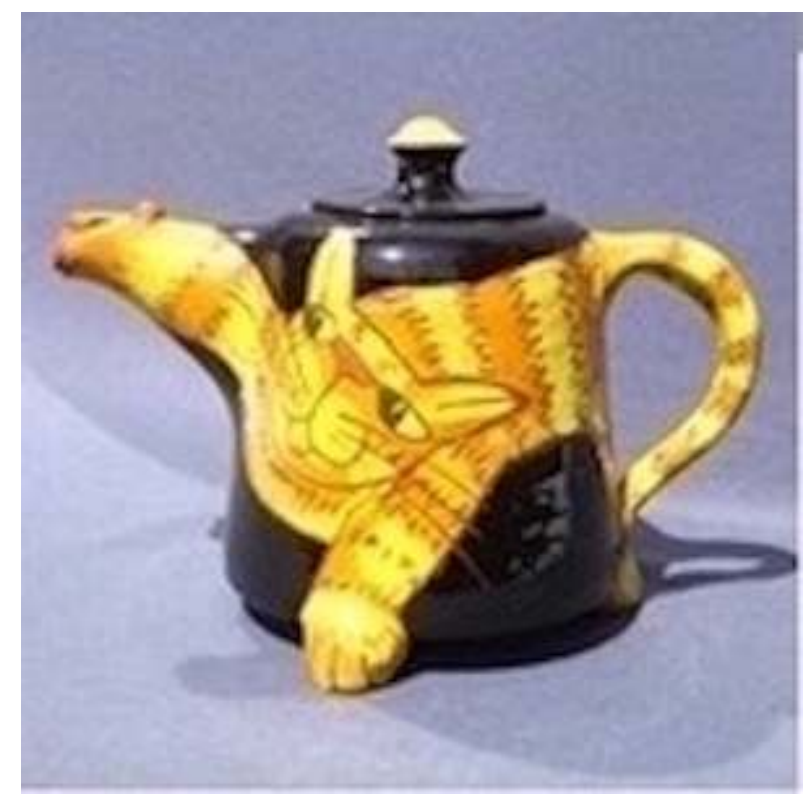

Photographie 8.

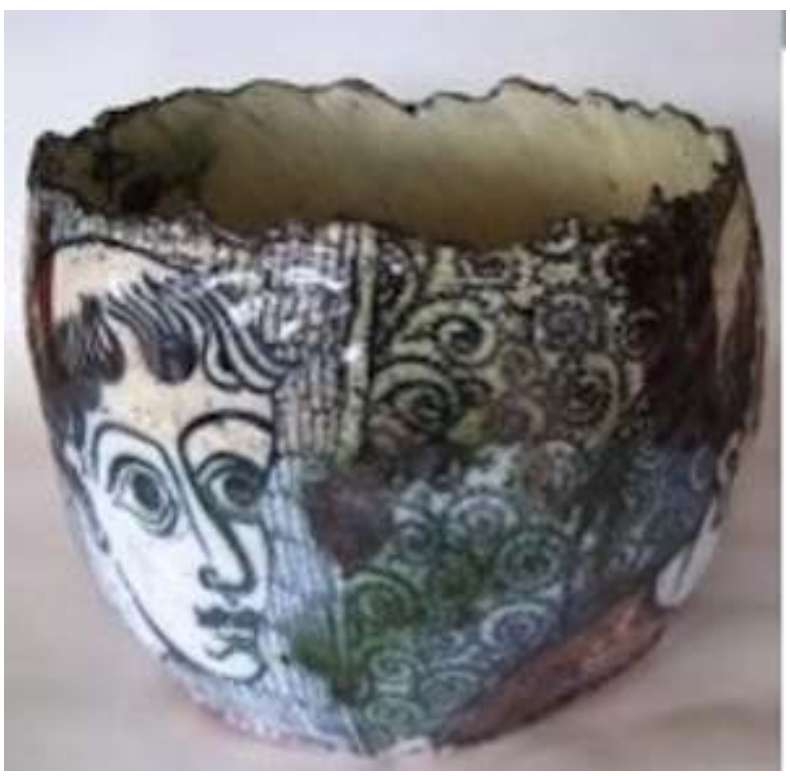


Photographie 9.

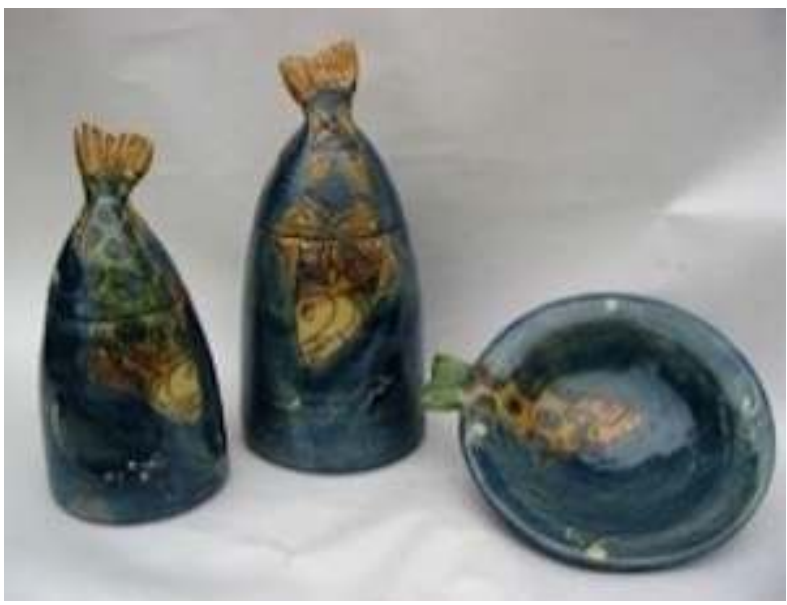

Photographie 10.

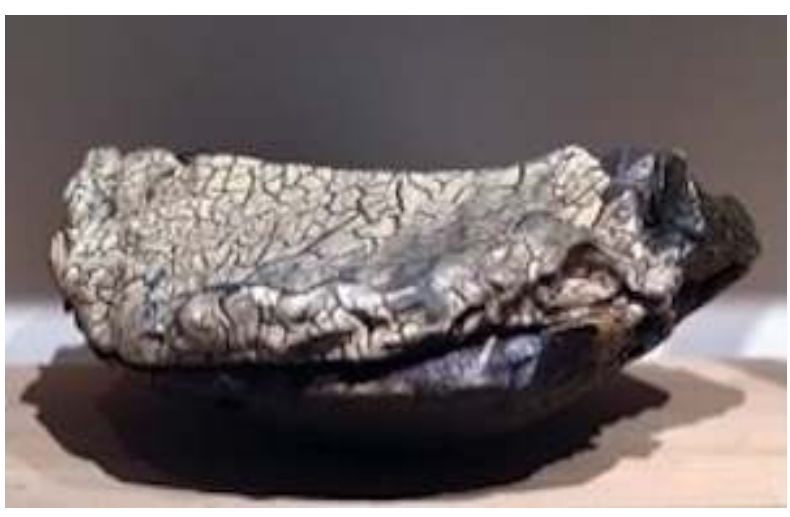

Photographie 11.

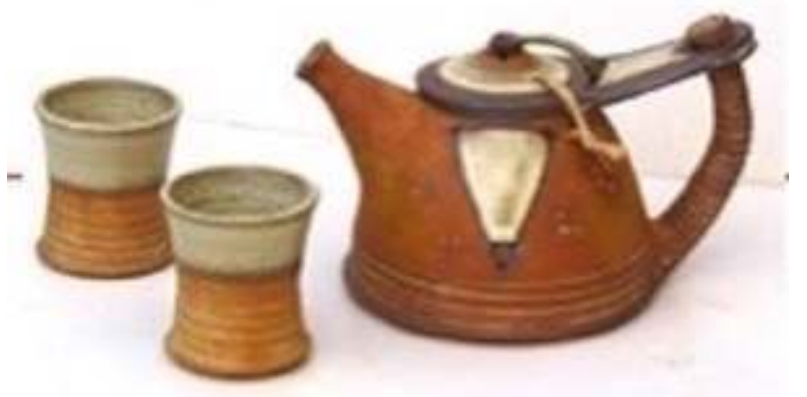


Photographie 12.

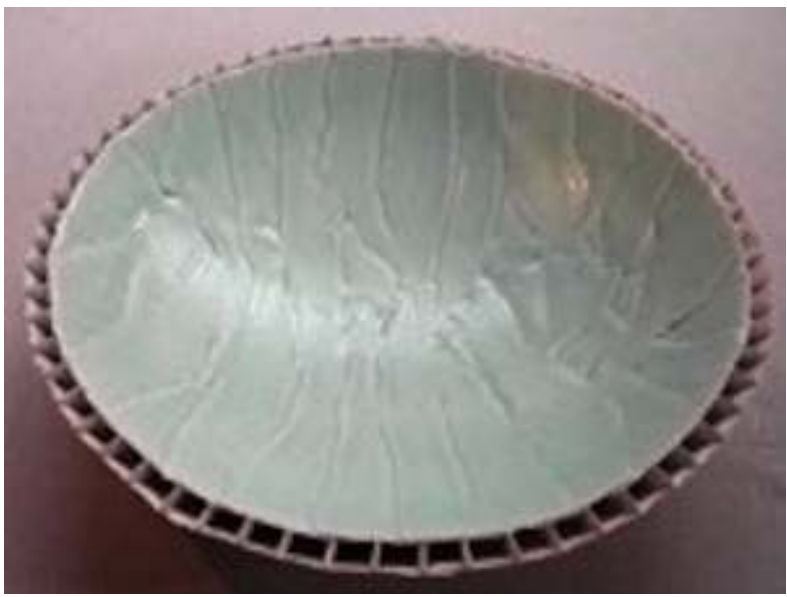

Photographie 13.

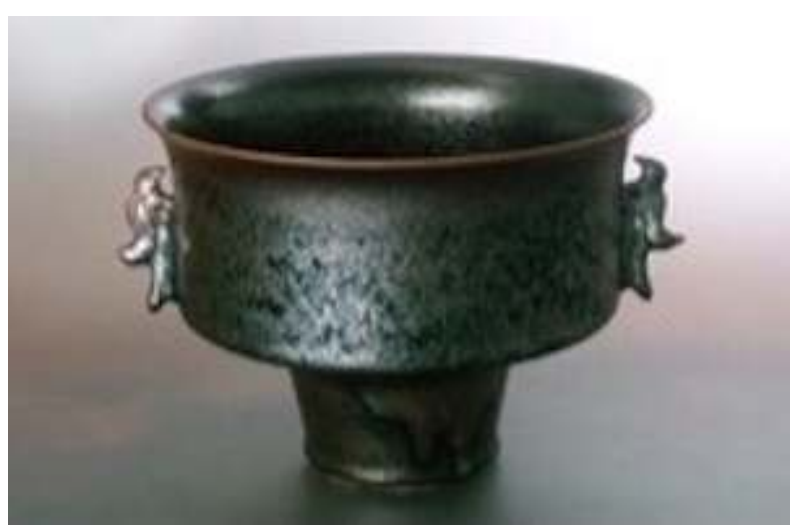

Photographie 14.

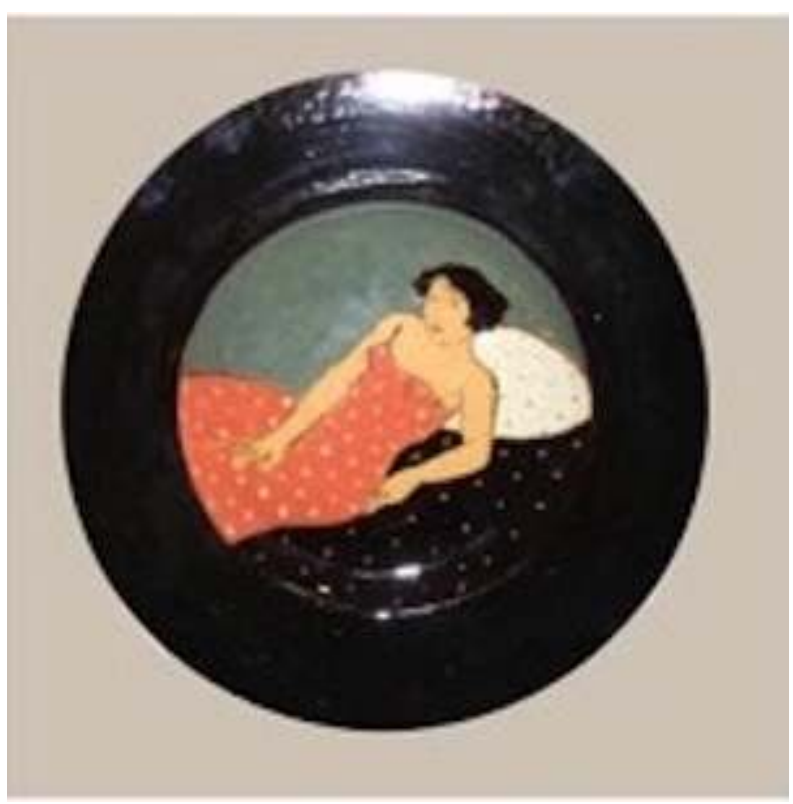


Photographie 15.

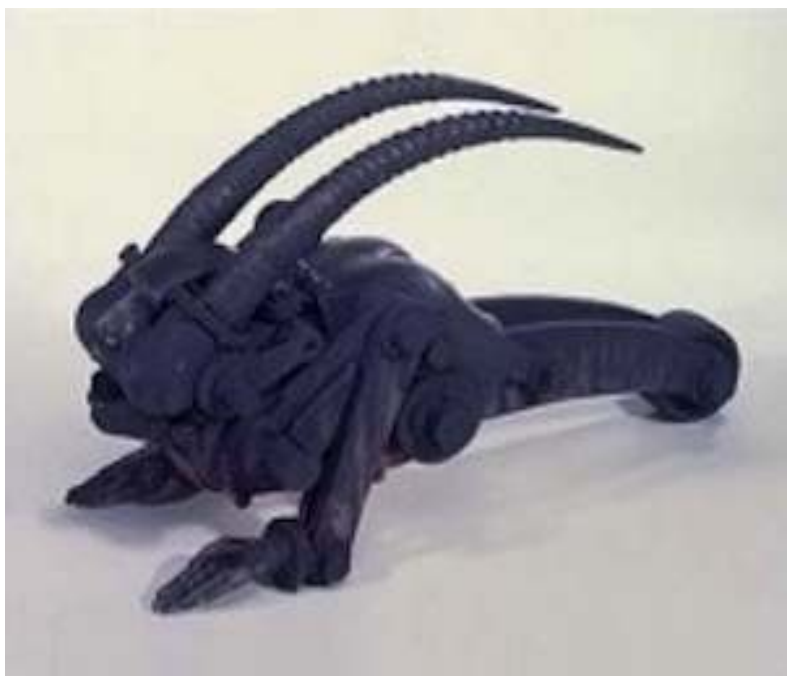

Corpus 3. Images (photographies 16, 17, 18, 19) utilisées lors des restitutions plus tardives, incluant une photographie de lieu, et comportant à chaque fois un commentaire.

Photographie 16.

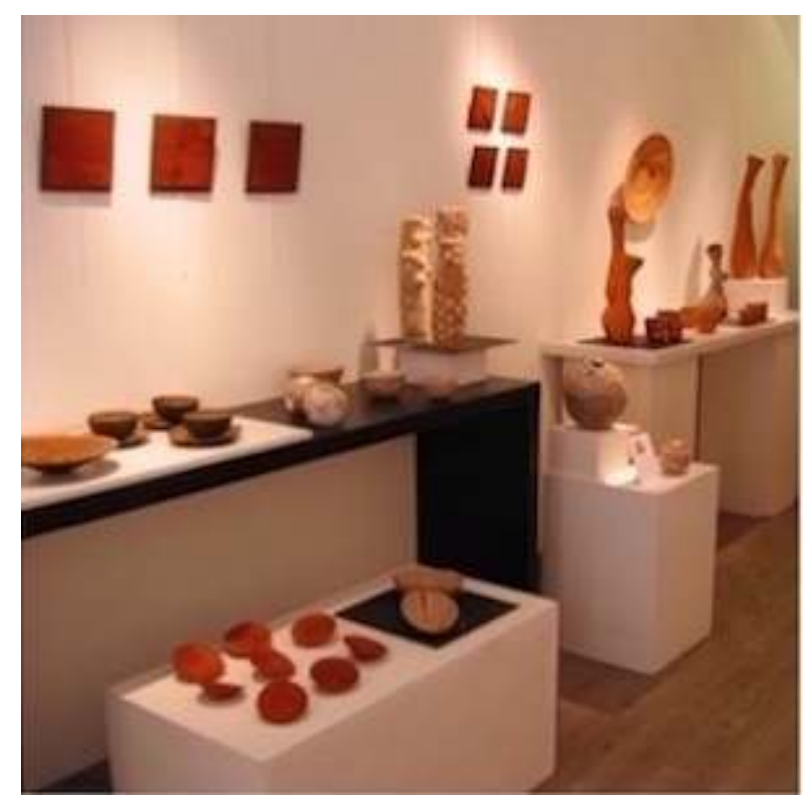

Exposition en galerie d'un collectif de femmes céramistes faisant pour la plupart des objets « utiles». 
Photographie 17.

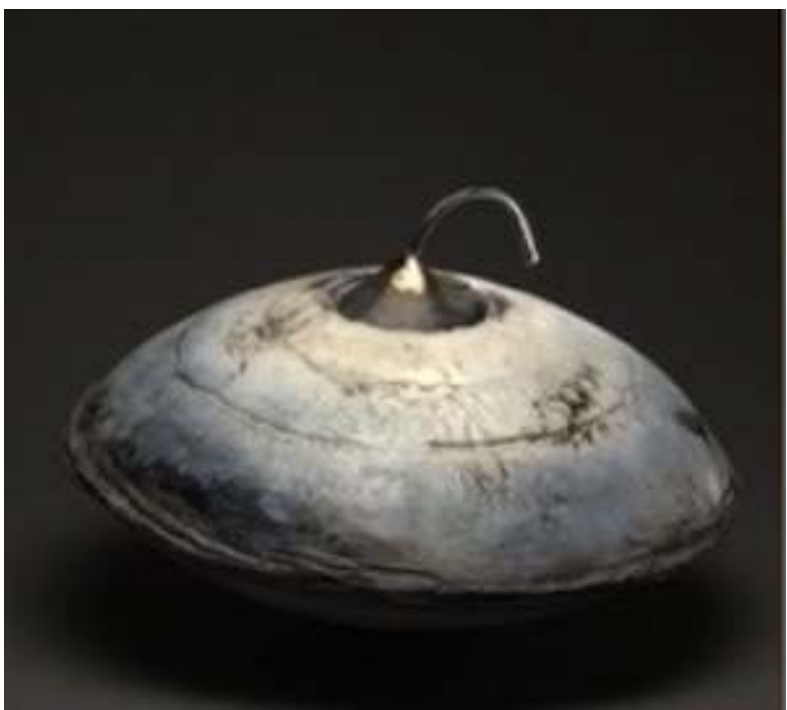

Céramiste très connue exposant ses sculptures... sur des « marchés de potiers » (env. $50 \mathrm{~cm}$ de diamètre).

\section{Photographie 18.}

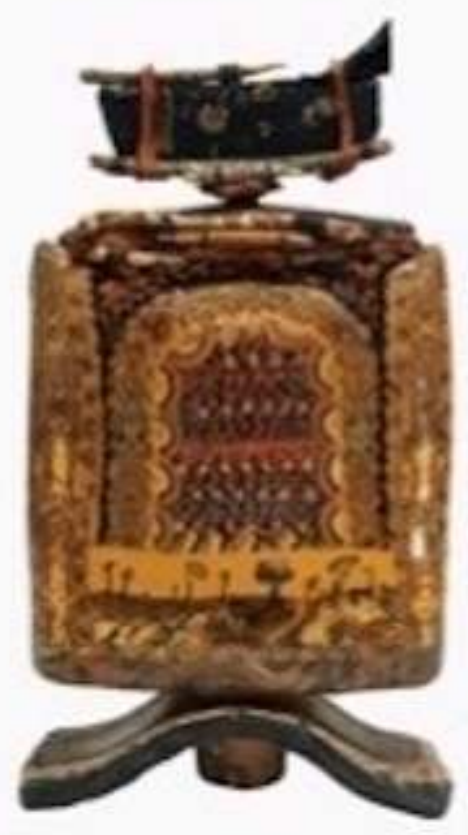

Boîte (env. $15 \mathrm{~cm}$ de haut) 
Photographie 19.

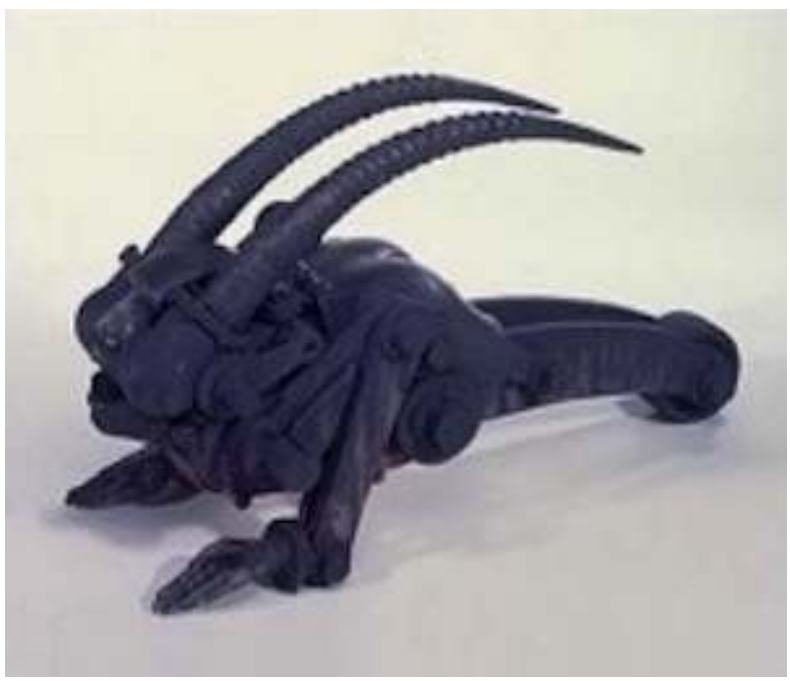

Sculpture $(70 \times 35 \times 40 \mathrm{~cm}$ de diamètre).

Plus tard, lors de la phase de rédaction de ma thèse, j'ai inséré un volet « illustrations » dans les Annexes correspondant à différents chapitres du manuscrit. Une page renvoyant par exemple aux chapitres 1 et 2 illustre la naissance de la céramique d'art au XXe siècle à partir des supports suivants: une carte postale ancienne, et la reproduction de différentes pièces et outils de céramistes du début du siècle ou contemporains, toutes trouvées sur internet.

Corpus 4. Images (photographies 20, 21 et 22) regroupées sur une page des Annexes illustrant les parties historiques de la thèse, réalisée à partir d'images trouvées sur internet et commentées succinctement :

Photographie 20. La production imagière (céramique utilitaire populaire singularisée par des éléments figuratifs). 


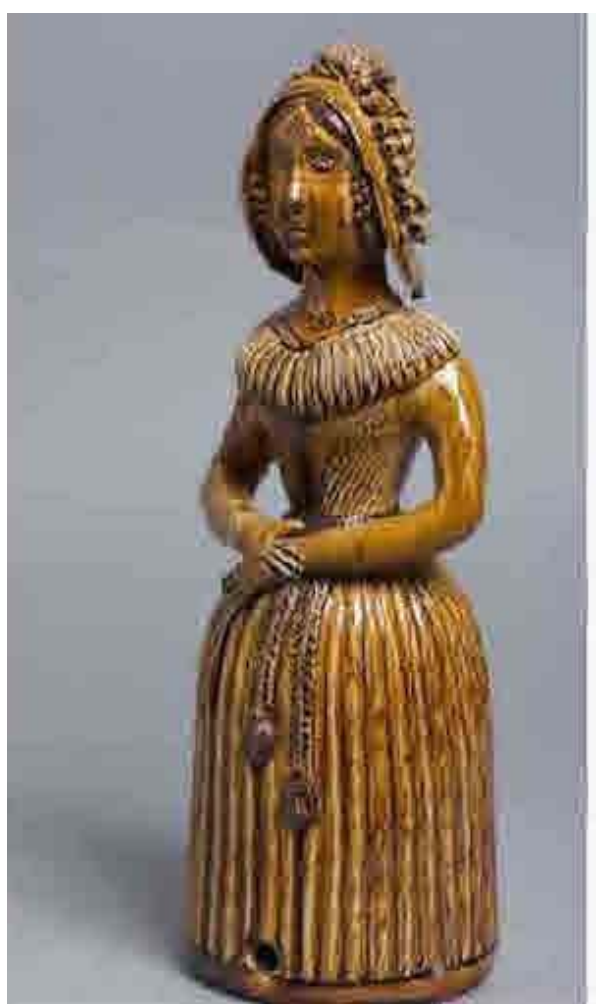

Une pièce de Marie Talbot à la Borne (fin XIXe).

Base « Joconde » (Portail des collections des musées de France) : 
Photographie 21. Un vase de Clément Massier établit à Vallauris à partir des années 1880.

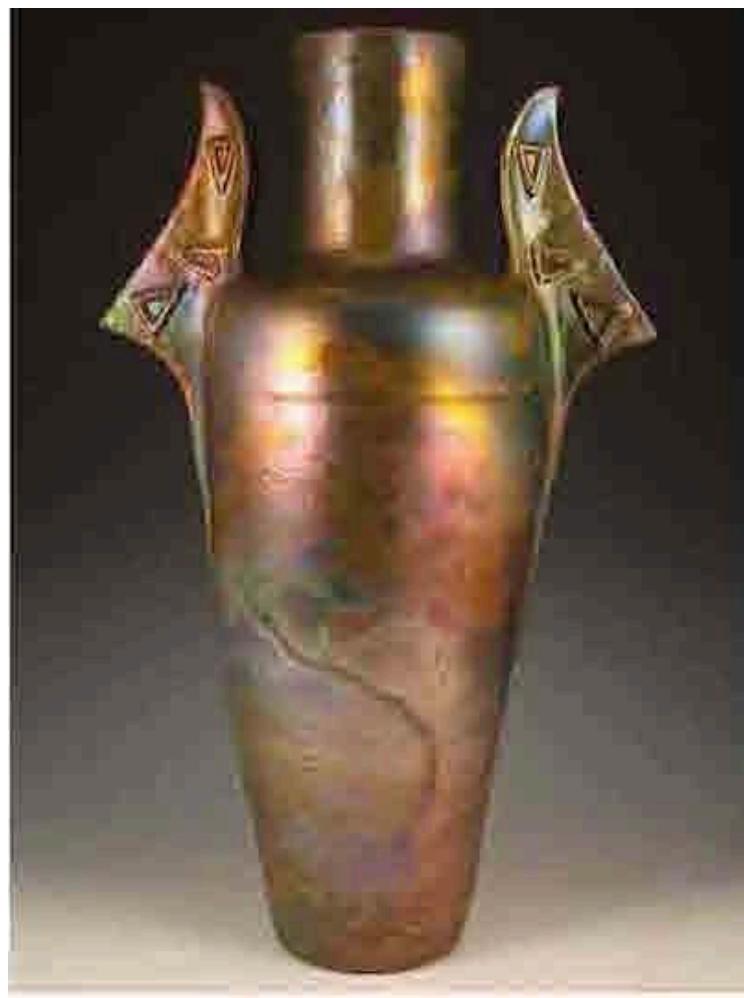

Ses plats en faillance émaillée, ornés de fresques figuratives et paysagères aux lustres métalliques témoignent des expérimentations artistiques bien avant l'apparition massive des céramistes d'art. Base « Joconde » (Portail des collections des musées de France) :

Photographie 22. Une poterie en 1908.

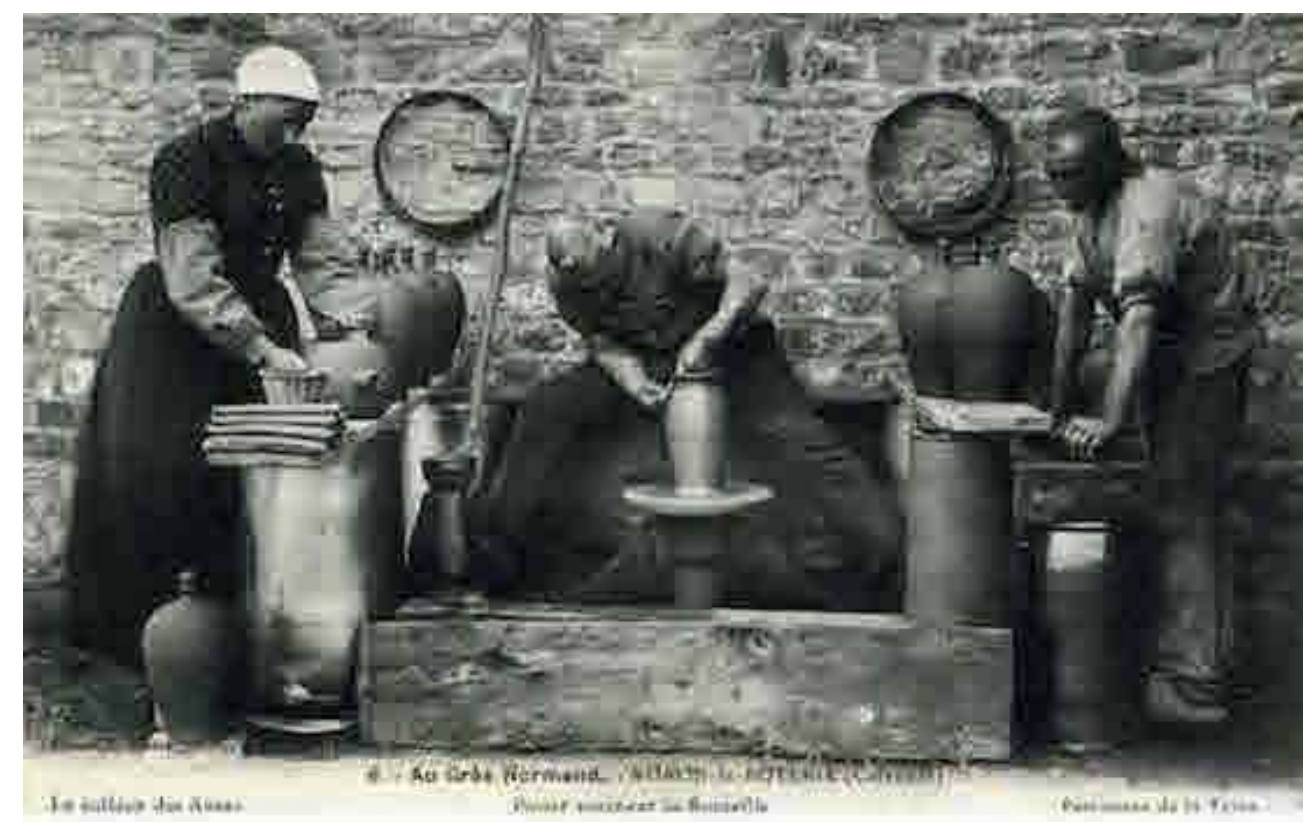

La poseuse d'anse (habilleuse de pots), son mari le tourneur et à droite, le batteu qui prépare les pains d'argile pour le tourneur. 
16 J'ai également inséré des photos de fours à bois construits par des enquêtés, prises au cours des phases d'enquête ( $c f$. images présentées dans le corpus 1). L'absence d'image, puis cette présentation de quelques images en guise d'illustration ont généré des effets indirects : les réactions qu'elles ont suscité parmi la communauté de chercheurs ont agi comme facilitateur de l'analyse et révélateur de certaines caractéristiques de mon objet, d'une part, et ont permis de produire un sens commun mobilisable à la fois comme matériau empirique à part entière, d'autre part.

Un premier enseignement tiré de la quasi absence d'images dans la restitution de mon travail au début de ma thèse émane des commentaires avides d'images, ou regrettant qu'il n'y en ait pas. Ces derniers témoignaient de la place de cet objet dans la hiérarchie des biens scientifiques, et plus généralement de sa dimension atypique. En effet, si j'ai mis des images dans mes présentations power point, c'est d'abord en réaction à la difficulté à discuter collectivement au sein du groupe de pairs de l'activité professionnelle que j'étudiais. Lors de certains colloques, le temps de discussion imparti à ma communication pouvait en effet être quasiment monopolisé par ce que j'appelais à l'époque des "questions de curiosité ». Il s'agissait de commentaires ou interrogations portant sur des aspects anecdotiques du métier, ou du moins étrangers à la problématique que j'exposais dans mon intervention. Ces interventions étaient généralement posées par un public peu averti, donc intrigué, et de ce fait parfois moins préoccupé par les aspects sociologiques et les montées en généralité possibles que par les détails de mon objet. Montrer des images du groupe professionnel étudié visait donc à réduire le risque de déviation de la discussion scientifique sur ce type de questions. Cette aspect renvoie aussi au statut des objets, plus ou moins légitimes (quoi qu'en disent les discours sur le fait qu'il n'y a pas de "petits objets») et plus ou moins facilement appropriables par un auditoire profane.

Ensuite, cet objet mal connu, voire méconnu, apparaissait souvent aux profanes et à l'auditoire comme un " métier d'antan » (associé à la figure du potier cohabitant avec le maréchal ferrant et le forgeron du village). Or, je parlais de ce métier comme d'une profession récente, apparue au XXe siècle. La demande d'images traduisait ainsi, à mon sens, la difficulté pour un auditoire à se représenter une activité professionnelle aux caractéristiques a priori contradictoires entre elles : puisant à la fois dans des savoirfaire anciens, voire millénaires, tout en les dotant d'une signification nouvelle (Bajard, 2014), ou encore, réunissant des logiques propres à deux catégories institutionnellement et culturellement dissociées en France, l'art et l'artisanat. Dans ces moments là apparaissait avec force le fait qu'il s'agissait d'un métier nouveau, souvent méconnu, et aux normes particulièrement dissonantes avec les représentations ordinaires des productions culturelles. Or, ces aspects étant pour moi des évidences, je ne les avais pas totalement perçus avant cette confrontation à des tiers : l'observation des réactions d'un public profane face à mes photos a grandement facilité la prise de distance avec mon objet, puisque j'effectuais un enquête par « distanciation » et non par « dépaysement » (Beaud et Weber, 2010).

Enfin, après que j'ai commencé à présenter des images, la confrontation des illustrations à des tiers ( $c f$. images présentées dans les corpus 2 et 3 ) a, en plus de leur potentiel réflexif, eu pour effet de faire apparaitre des perceptions sociales mobilisables comme matériau empirique à part entière. Par exemple, les commentaires et questions posées par les universitaires indiquaient souvent chez ces derniers une distinction toute «naturelle ", du moins logique, entre artiste et artisan, galeries d'art et marchés 
de potiers. Or, la contestation de ces dichotomies est au coeur des normes professionnelles prônées au sein du groupe des céramistes d'art. J'ai donc fait un usage analytique de ces réactions, en considérant par exemple que les questions posées par des pairs sur ces photographies traduisaient un sens commun sur la céramique tout aussi intéressant à étudier que des coupures de presse ou des propos de visiteurs sur un marché de potiers.

\section{L'iconographie comme pratique sociale : l'enquête escamotée par le contournement d'une analyse interne}

Certaines images utilisées dans ma thèse ont été produites par les enquêtés eux-mêmes. Autrement dit, elles constituent des pratiques sociales au même titre que des prises de parole publiques, des manifestes, des manières d'exposer le travail ; je les ai étudiées pendant l'enquête, puis pendant l'écriture. Cependant, cette étude ne fut que partielle, escamotant une source possible de production de données.

\section{Saisir (ou pas) le potentiel des images en tant que parties d'un « tout »}

Lorsque j'ai intégré les images produites par les enquêtés eux-mêmes, il s'agissait de supports événementiels (affiches de marchés, cartons d'invitation à des expositions...) ou de photographies représentant les enquêtés et leur travail ou cadre de vie, accompagnant des portraits sociologiques. Je les ai insérées dans le manuscrit avant tout dans un souci de simplification de l'écriture : le but était de ne pas décrire un matériau empirique que je pouvais présenter directement au lecteur. Une mise en abyme de l'image - décrire une image par le texte - aurait en effet pu sembler absurde. Insérer des images contribuait ainsi à étoffer mon corpus empirique et mes analyses dans une démarche d'administration de la preuve, de cumulativité et de saturation des hypothèses. Pourtant, cet usage peut être qualifié d'inachevé, en raison d'un manque d'approfondissement de l'analyse interne de l'iconographie.

L'image comme pratique sociale fut mobilisée à divers endroits des Annexes. D’abord, plusieurs pages comportent des affiches d'événements. On y voit la diversité des visuels existant au sein du groupe professionnel pour annoncer un marché de potiers: le contenu de ces affiches illustre des programmations à géométrie variable, certaines étant minimalistes (le marché), d'autres comportant des animations, projections, conférences, etc. Surtout, c'est la variabilité des légitimités et du prestige au sein du métier que leurs organisateurs prétendent conférer à leur événement qui est donnée à voir. En effet, certaines affiches au graphisme épuré et sophistiqué, aux photos travaillées, sont à l'image de la place de l'événement dans la hiérarchie interne au métier : il s'agit de « beaux » ou de " grands » marchés. D’autres, presque monocolores, mentionnant un simple dessin et annonçant l'événement dans une police démodée, correspondent à la taille et à la réputation des petits marchés de potiers peu connus. L'analyse interne de ces affiches, indispensable au même titre que l'analyse de discours, apparaît furtivement dans le corps de la thèse: je la rattachais alors à d'autres pratiques professionnelles de présentation de soi et de régulation interne du métier. 
23 En plus de ces documents, j'ai également réalisé dans les Annexes cinq portraits sociologiques (environ 10000 signes chacun) incluant à chaque fois quatre à cinq images.

Corpus 5. Images (affiches 1, 2 et 3) présentées sur une page des annexes montrant des iconographies comme pratiques communicationnelles (celles des organisateurs de «beaux marchés ») : celles-ci sont commentées et renvoient à des passages précis du manuscrit, ainsi qu'à des enquêtés.

Affiche 1. Une invitation pour un marché réputé, ceux que les professionnels qualifient de « beau marché ».

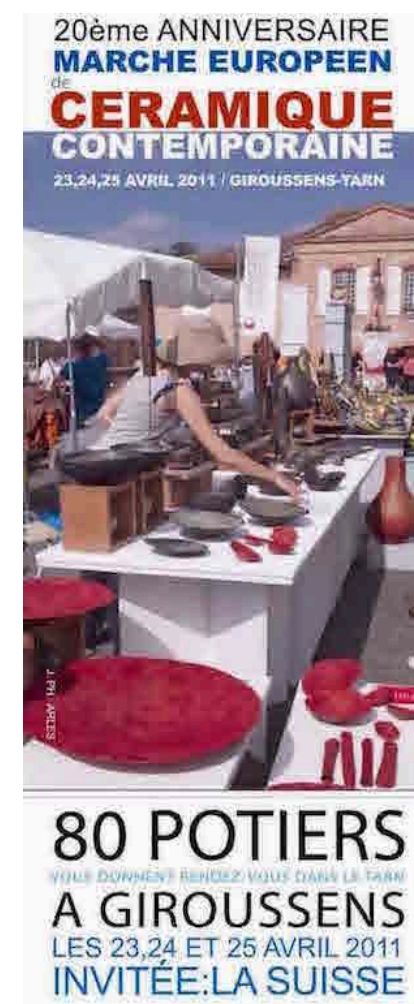

Au premier plan, le stand de Camille, cette céramiste incarnant ces « figures » qui en ancrant leurs pratiques dans le Métier. 
Affiche 2. Le marché de Dieulefit (avec un pays invité cette année-là : l'Australie), également reconnu.

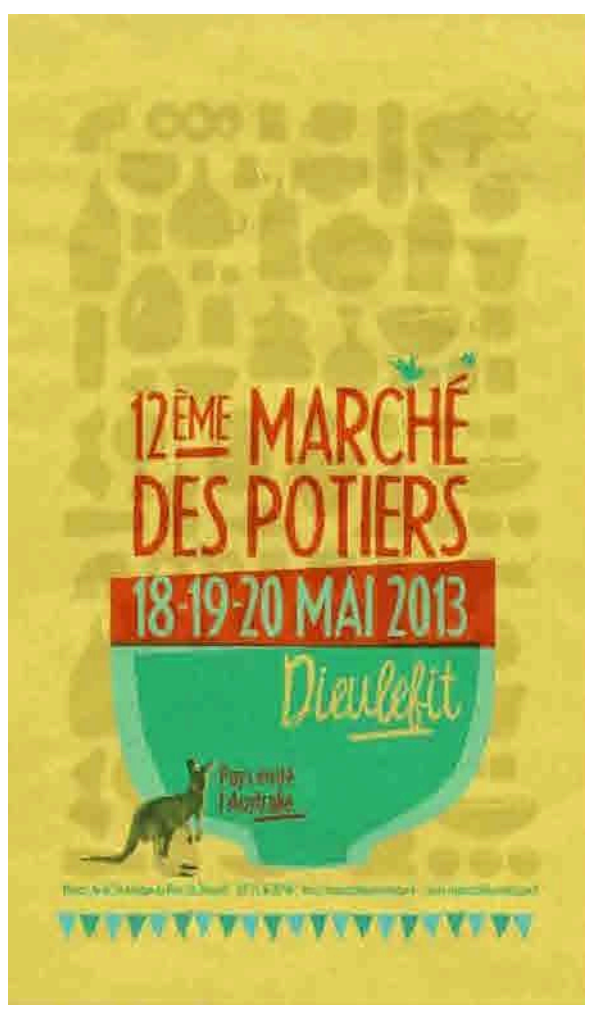


Affiche 3. Le marché de Tupiniers à Lyon fondé en 1986.

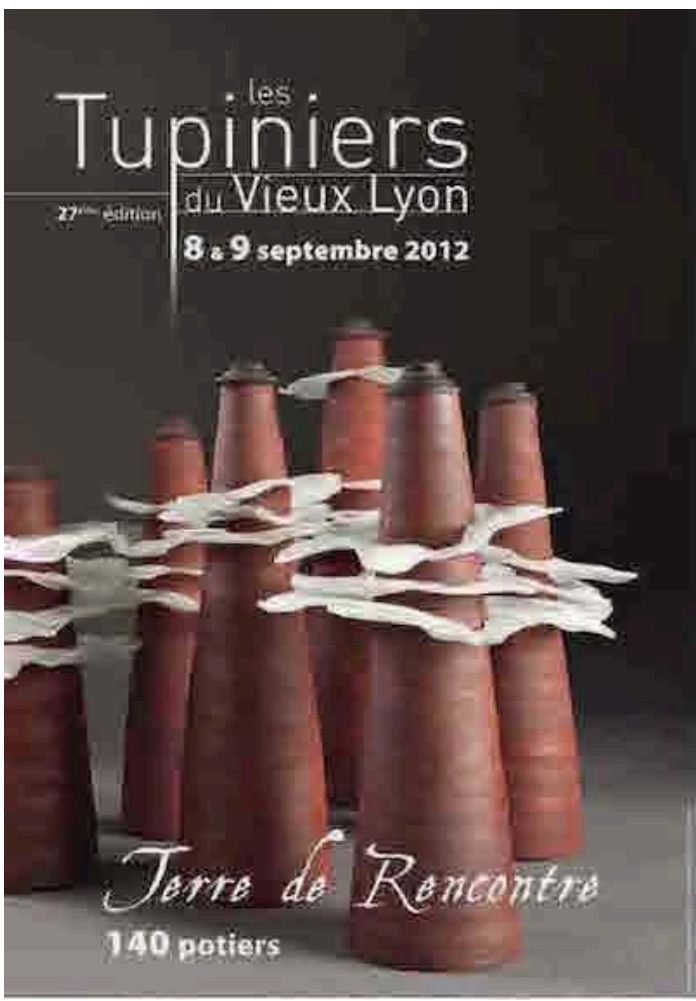

L'affiche présente d'un travail coéralisé par un céramiste et un maître-verrier : en plus de marché de plein air sur la place Saint-Jean accueillant 140 céramistes, une exposition thématique est organisée (cette année-là, «Duos » : étaient exposées les pièces coréalisées par un ou une céramiste et un autre artiste - verrier, calligraphe et dessinateur de bandes-dessinées).

Corpus 6. Images présentées sur la dernière page d'un portrait ; les photos, issues d'un internet et de mon carnet de terrain, donnent à voir l'enquêté en train de travailler, son lieu de vie et de travail, et quelques unes de ses pièces : 
Photographie 23.

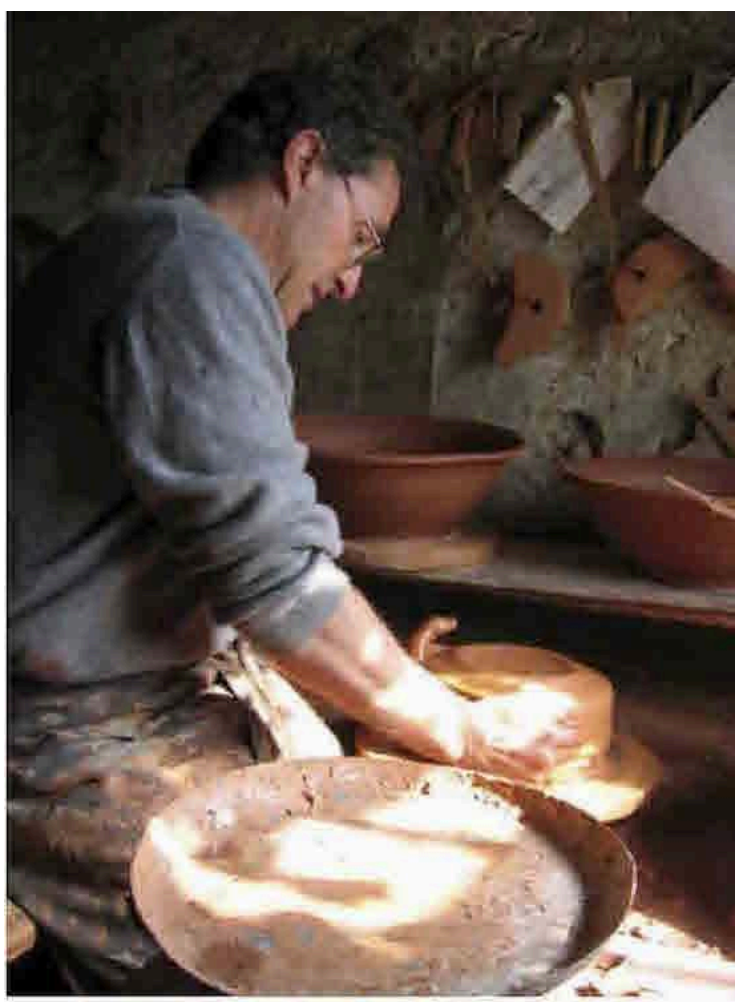

Christian devant son tour à pied.

Photographie 24.

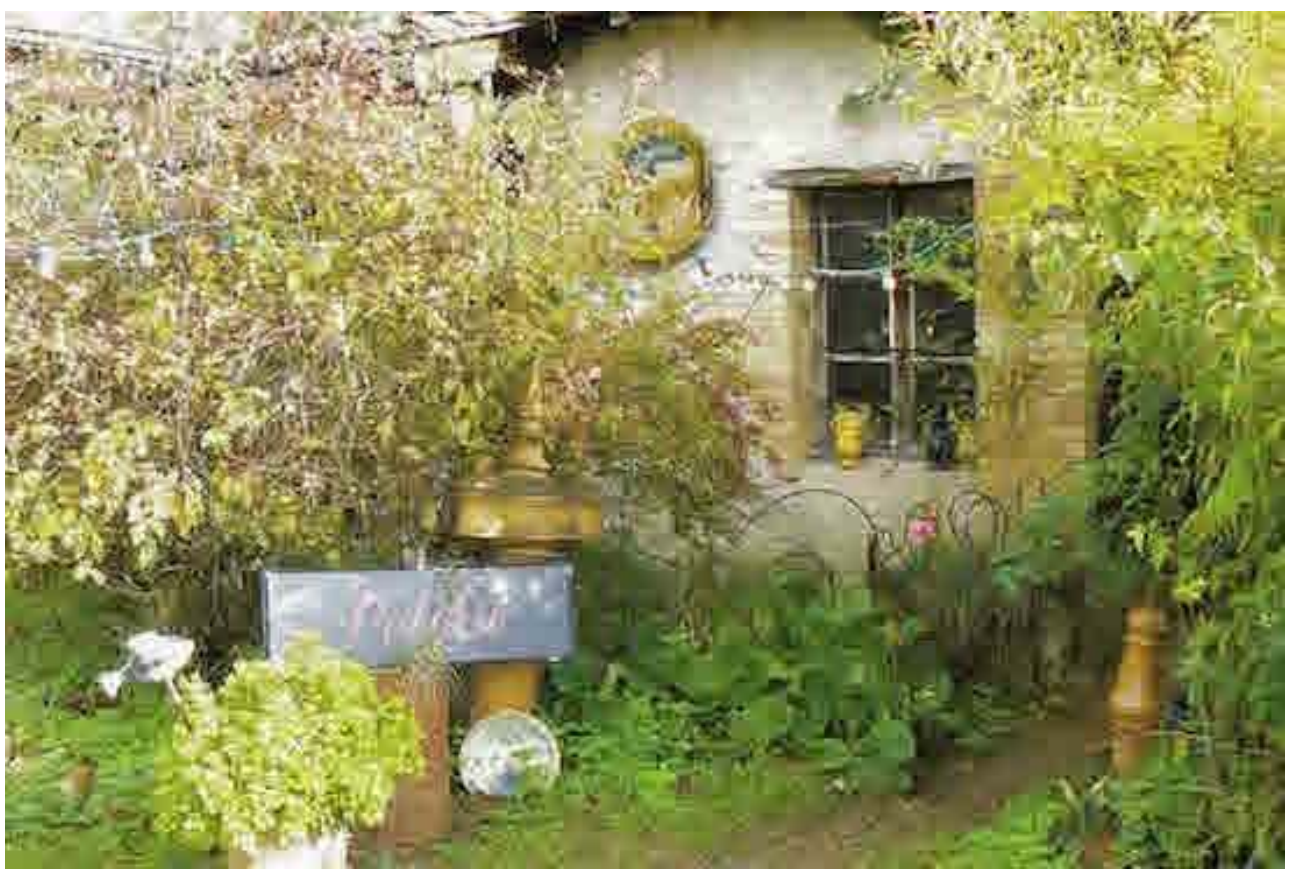

La porte d'entrée de son atelier et son jardin agrémenté d'objets en céramique, visible dès l'arrive au domicile. 
Photographie 25.

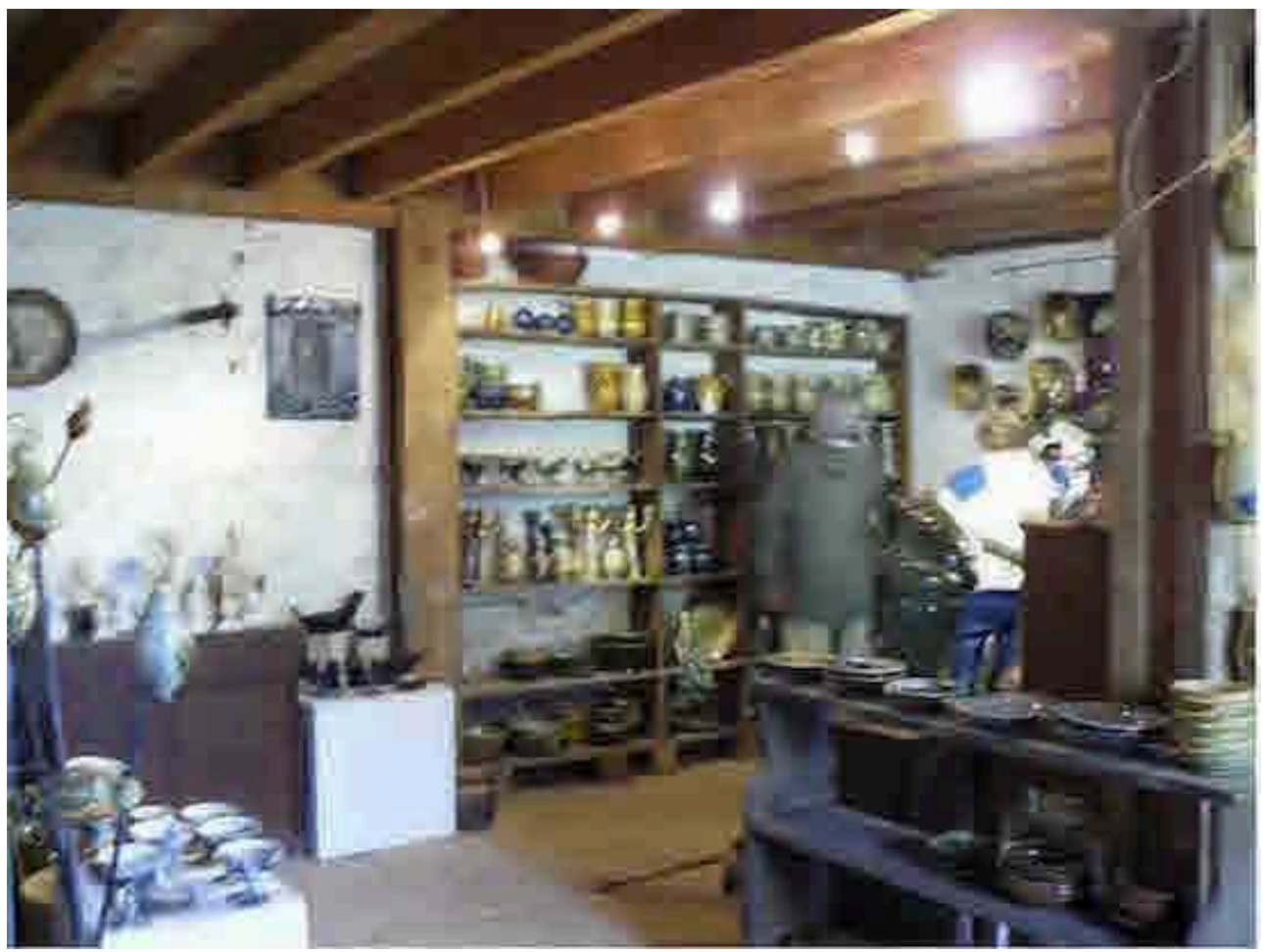

La boutique attenant à l'atelier, où Christian et sa compagne vendent leur travail.

\section{Photographie 26.}

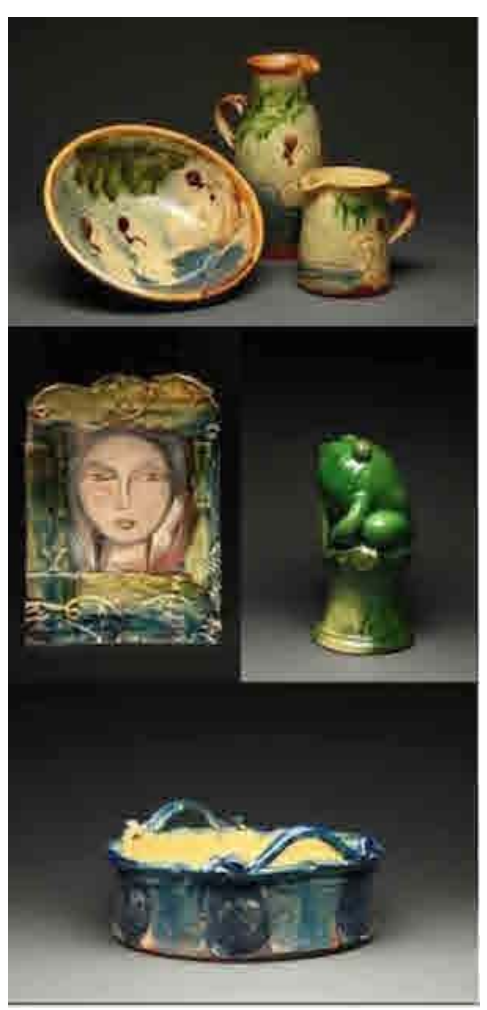

Quelques unes de leurs productions en terre vernissée, en majorité utilitaire. Site de l'association régionale D'Argile dans laquelle ils sont adhérents. 
Ces portraits de céramistes visaient à réunir différents aspects traités tout au long du manuscrit, et à montrer comment ceux-ci s'imbriquent dans la trajectoire d'un même individu. Dans une approche "totalisante ", chaque portrait restitue donc une pluralité de dimensions - esthétiques, politiques, commerciales, vie familiale - des carrières et conditions d'existence de l'enquêté. À ces portraits étaient associés plusieurs images représentant les enquêtés ou leur environnement de travail: photographies issues d'articles de presse ou, beaucoup plus fréquemment, de leurs propres supports de communication personnels (blogs, sites internet...). Voici comment je présentais cette démarche :

"il s'agit, de cette façon, d'offrir au lecteur des repères concrets permettant d'associer des images aux éléments esthétiques, graphiques, techniques, ou même vestimentaires ici décrits. En outre, s'il est apparu utile de proposer des images de quelques objets en céramique, il nous est apparu plus signifiant sociologiquement de proposer ces repères iconographiques de manière située et relationnelle, accompagnant ces portraits, plutôt que de manière désincarnée et isolée dans une autre partie des Annexes. »

25 J'ai, ce faisant, réalisé une démarche proche de la " mise en série photographique ${ }^{8}$ » que font certains photographes, qui façonnent les contours de «types sociaux» en s'intéressant à des cas singuliers (Maresca, 2011) ${ }^{9}$. Ici, le cheminement fut seulement différent dans la temporalité, puisque mes analyses et montées en généralités étaient déjà constituées avant de recourir à l'insertion des données visuelles lors de la rédaction des Annexes ${ }^{10}$.

Dans les deux cas - les images d'affiches, et les images liées aux portraits - j'explicitai dès la rédaction que leur sens ne pouvait apparaître que relationnellement. Pourtant, l'analyse interne a souvent été réalisée de manière tout à fait inconsciente et peu approfondie. Je me contentais en effet de mettre en relation les images avec d'autres éléments : la pente d'une trajectoire sociale, des ressources culturelles, des pratiques artistiques et scénographiques. Autrement dit, le sens de ces images était implicite: soit il était supposé apparaître par leur simple association à d'autres caractéristiques, soit ces images ne venaient qu'étayer, illustrer, confirmer ces caractéristiques. Ce passage sous silence témoigne d'un implicite fortement ancré au moment de la rédaction: les images n'étaient qu'un élément parmi d'autres pratiques sociales formant un "tout cohérent». Si je me suis davantage attachée à analyser l'hexis corporelle, ou l'ambiance des lieux observés (par exemple), on constate un relatif vide concernant l'analyse du contenu des images. Même s'il n'a pas remis en cause l'analyse, cet usage que l'on pourrait qualifier d' « inachevé » de l'iconographie est révélateur du potentiel inexploité de ce type de matériaux. On peut reproduire le même constat concernant l'usage des images au sein de la relation d'enquête.

\section{Les images des travailleurs : une utilisation parcellaire dans la relation d'enquête}

Lorsque j'allais rencontrer un.e enquêté.e, je recherchais généralement des informations préalables sur celui ou celle-ci ${ }^{11}$, et consultais notamment son site web lorsqu'il ou elle en possédait un, véritables outils de travail sur le plan commercial. Par ailleurs, j'avais inclus dans ma grille d'entretien la thématique de « la communication », et demandais donc systématiquement aux enquêtés comment ils réalisaient leur publicité, s'ils montraient leur travail sur internet, et enfin, quelle partie du travail ils 
donnaient à voir ${ }^{12}$ à autrui : s'agissait-il du processus (les gestes, les étapes de la réalisation des pièces, en somme, les « coulisses du travail », voire leur lieu de vie), ou du seul résultat (les objets fabriqués)? La question de l'image d'un point de vue indigène, c'est-à-dire ce que l'on donne à voir de soi et de son travail, était donc clairement systématisée dans l'enquête. Premièrement, ce qui m'intéressait à l'époque dans ces supports visuels n'était pas tant leur contenu en soi, que le rapport que les enquêtés entretenaient à l'égard de ceux-ci, et donc de leur propre image. En effet, " étudier le travail, ce n'est pas seulement regarder les travailleurs, c'est regarder avec eux. Et aussi essayer de regarder leurs regards » (Meyer, 2014, 148). En tant qu'outils de travail, ces images montraient ce qu'est - ou ce que donne à voir - un enquêté, mais aussi ce que cette personne tente d'être ou de devenir face à ses pairs ou sa clientèle. Deuxièmement, ces supports visuels étaient au service d'une dimension en partie déniée du travail : le savoir être, la présentation de soi, voire la "publicité » (terme dévalorisant pour beaucoup de ces artistes et artisans qui prétendent vendre autre chose que de simples objets, et en grande partie rassemblés autour de la dénégation de l'économie monétaire (Bourdieu, 1993b)). À travers ces deux séries d'éléments, on voit que comprendre le rapport que les enquêtés entretenaient à ces outils de communication et à leur contenu me permettait ainsi d'ouvrir l'analyse dans de multiples directions du point de vue de la sociologie du travail: la gestion du «sale boulot " (Hughes, 1996), puisque réaliser ces visuels est généralement peu apprécié par les professionnels et considéré comme une difficulté et/ou une perte de temps ; la disposition - capacité et appétence - à mettre son travail en récit et à le doter d'une " plus-value symbolique ${ }^{13}$ "; leur manière de considérer les objets qu'ils fabriquent et de les mettre en scène (photographiés comme des pièces uniques en galerie d'art, ou au contraire donnés à voir sous forme d'inventaire dans un dispositif beaucoup moins " artifié », etc.) ${ }^{14}$. Or, si j'ai étudié ce type de relation entre les enquêtés et leur image dans le vase clos de mon bureau, la photo-elicitation n'a pas fait partie du dispositif, tout simplement parce que je n'en avais pas eu l'idée. C'est là encore le signe de cette inclusion encore très inachevée de l'image dans l'appareillage de méthodes qualitatives ou quantitatives en sciences sociales. Cela tient sans doute au fait que j'ai calqué mon rapport à ce type de données sur l'utilisation que je faisais des données langagières : je ne confrontais jamais les enquêtés avec les analyses de leurs propres discours (hésitations, lexique utilisé, etc.), car cela aurait sans doute porté la violence symbolique de l'entretien à son paroxysme. Et sans réellement m'en rendre compte, il en fut de même pour l'image: lorsqu'il s'agissait de discuter autour des supports de communication personnels des enquêtés, je n'ai que très rarement abordé l'analyse interne ou sémiotique de ces images avec eux ne serait-ce que sur le registre du commentaire («Pourquoi avoir choisi telle photo? Que dit-elle selon vous?»). Je me suis en effet contentée d'une mise en relation rapide de leur contenu avec les discours qu'ils y associaient. En fait, nous pouvons là aussi parler d'exploitation inachevée ou parcellaire de l'image comme pratique sociale : je m'en suis tenue à des discussions sur la communication et l'iconographie dans leur principe et dans leur mise en œuvre, en tant que pratique sociale et professionnelle inscrite dans l'ensemble d'un faisceau de tâches. Cela n'a pas constitué en soi un problème dans mes analyse, mais indique en revanche $a$ posteriori ce que l'on a osé (ou pas) aborder avec les enquêtés, ainsi que le potentiel (ici partiellement inexploité) de ces données. 


\section{Montrer de « belles images » ? Dilemmes autour de l'esthétique} une démarche univoque : il ne suffit pas d'opter pour ce matériau, encore faut-il aussi se demander de quel matériau ou pratique photographique il s'agit, et ce que l'attrait pour des images plus ou moins esthétisées dit de notre rapport à l'objet.

\section{Quand nos valeurs s'enchevêtrent dans l'image : neutralité axiologique et partis pris sociologiques}

À partir du moment où j'ai introduit des images dans certains supports de communication restituant les résultats de mon travail, j'ai surtout utilisé des planches d'illustrations de différents objets produits par les céramistes (plus rarement, des illustrations des scénographies de vente) (cf. images présentées dans les corpus 2 et 3 ). La diversité des objets photographiés visait à illustrer l'éclectisme des pièces produites et des techniques employées : céramique utilitaire et sculpture, matériaux et savoirfaire diversifiés, prix variables, exposition en galerie ou sur un marché de plein air. Plusieurs réactions émanant d'universitaires écoutant ma communication portaient alors sur la manière dont $\mathrm{j}$ 'avais présenté ces pièces : sur les premières présentations réalisées au début de ma thèse ( $c f$. images présentées dans le corpus 2 ) chacune d'elle figurait de manière singulière, la plupart du temps sur un fond uni, c'est-à-dire scénographiées comme des pièces uniques ou du moins, de manière artifiée (Heinich et Shapiro, 2012) ou ennoblie.

Certes, ce choix tenait à des questions prosaïques de visibilité des images (un fond complexe rendait difficile la perception de photos de pièces), ainsi qu'au stock disponible sur internet: il était beaucoup plus facile de trouver des photos de pièces photographiées sur un fond neutre, bien éclairées, que des pièces photographiées dans un environnement ordinaire. Toujours est-il que j'avais dans ces circonstances mélangé inconsciemment plusieurs statuts de l'image (Verdalle et Israël, 2010, 10) : l'image comme matériau "donné », et l'image comme résultat d'une "mise en scène ». Ces diapositives, qui provenaient de différentes sources ${ }^{15}$, étaient en effet des "donnés", au sens de matériau empirique et pratique sociale. En revanche, leur agrégation sur un nouveau support constituait une nouvelle "mise en scène ", réalisée par moi-même. Les remarques qui m'ont alors été faites en ces occasions ont questionné mon propre rapport à ces objets dans la mesure où mes sélections avaient inconsciemment reproduit une forme de valorisation du travail : j'avais mis en avant l'unicité des objets (donc un statut social de ceux-ci valorisé) et leur qualité (je présentais rarement des images de "mauvais céramistes", selon les critères indigènes). Les premières présentations - et représentations donc - de mes travaux furent donc le moment d'une première prise de conscience de ma relation à l'objet et aux valeurs prônées dans cet espace professionnel de la céramique d'art. Elles ont également alimenté une réflexion sur ma propension à travailler sur les "gagnants » du métier en termes de maintien dans l'emploi ou de réputation, par définition plus visibles que ceux qui « échouent ».

Images du travail, travail des images, 3 | 2017 


\section{L'image et la prose : hésitations d'une profane autour du « discours iconographique »}

31 J'ai voulu, lors de la phase de rédaction, insérer dans mon manuscrit des photos de Jérémy, le photographe évoqué plus tôt (cf. encadré). On peut facilement qualifier ses photographies - toutes en noir et blanc - de belles sur le plan esthétique, tandis que les miennes étaient parfois mal cadrées, sans aucun travail de l'image (a posteriori ou lors de la prise de vue), et réalisées avec un matériel photographique sommaire. À la question qui m'avait été posée à l'époque : " pourquoi veux-tu utiliser ces belles photos plutôt que les tiennes? ", j'avais plusieurs pistes de réponse. Premier motif : les images du photographe permettaient sans conteste de restituer des détails et facettes de mon terrain que mes prises de vue ne permettaient pas de voir: en raison de leur qualité technique, ces photographies mettaient en lumière - au sens propre et figuré - des gestes, des postures corporelles, ou encore des textures de matériau (par exemple, la souplesse de l'argile mouillée, ou encore la vivacité des escarbilles de bois incandescentes dans l'air lors d'une cuisson...). Second motif, la volonté de vouloir utiliser de «belles images " s'inscrivait, à l'époque de la rédaction de ma thèse, dans l'optique de vouloir rendre un manuscrit qui puisse être un « bel objet » : un travail que l'on aurait plaisir à feuilleter, à regarder, dont les illustrations renforceraient l'envie de lire les analyses qui y étaient liées. Il est possible que cette seconde raison fut liée à une troisième, beaucoup plus inconsciente : celle de vouloir mettre en lumière (au sens figuré uniquement cette fois-ci) le travail des céramistes. Toutefois, à l'époque de cette réflexion, j'étais en train de rédiger ma thèse, et la démarche de réflexivité réalisée pendant les années qui avaient précédé m'avaient, me semble-t-il, bien prémunie de toute intention de sublimation de mon objet d'étude. Finalement, j'ai graduellement modifié mon rapport aux images de Jérémy, pour finalement renoncer à les insérer dans le manuscrit final. C'est un questionnement esthétique, et en fait, axiologique, qui en est à l'origine : que racontent les photos?

La photographie revêt un statut intermédiaire : elle traduit "une vérité " (Becker, 2007) sans médiation (celle des mots), mais elle est dans le même temps un regard, une esthétique singulière (qui plus est, ancrée dans les mondes de l'art). Par ailleurs, sans même parler de falsifier une image, on sait combien il est aujourd'hui aisé pour tout un chacun de modifier un cadrage, une texture, des couleurs. Il n'est pas très difficile de conférer une atmosphère et des significations à travers le travail de l'esthétique photographique, même sommaire. Le risque étant notamment, on l'a vu plus tôt, de reproduire certaines catégories indigènes, en montant par exemple uniquement ce que les professionnels qualifient de "vrai boulot ", c'est-à-dire ce qui fait particulièrement sens à leurs yeux dans le travail (Bidet, 2011), ou de sublimer le métier dans ses instants les plus exceptionnels. C'est sans doute cet écueil qui m'a fait renoncer à l'usage des photographies de Jérémy : ses photos étaient travaillées de façon à mettre efficacement et joliment en évidence un fond (l'environnement de travail) ou au contraire un premier plan (les gestes et le corps du céramiste par exemple). Autrement dit, celles-ci me semblaient déjà trop investies d'un commentaire. Les images de Jérémy me semblaient déjà dire quelque chose qui ne m'appartenait pas, tandis qu'avec mes propres photographies, il me semblait que je produirais davantage des images que des idées (Maresca, 2007, 65). Maîtrisant mal les termes du débat convoquant l'image et la prose $^{16}$, j'ai finalement renoncé à utiliser les images de Jérémy : les quelques images 
présentes dans le corps du manuscrit ne sont venues qu'en appui des descriptions et analyses produites par l'écrit, que je maîtrisais mieux. J'avais en effet le sentiment de pouvoir mieux maîtriser mon cadrage et le récit ethnographique à travers l'écriture. Par ailleurs, la beauté formelle de ses images me semblait conforter un soupçon dont je cherchais à me prémunir plus généralement tout au long de mon travail de thèse : celui d'un rapport "enchanté » à mon objet d'étude, puisque j'entretenais des liens de familiarité avec celui-ci. C'était même ce soupçon qui m'avait conduite à écrire un article méthodologique dans lequel je défendais, à la suite des travaux réfutant la supposée neutralité du chercheur, un usage heuristique des "perturbations" habituellement considérées comme des biais (Bajard, 2013).

En réalité, c'est seulement a posteriori que j'ai mesuré combien la question de ce que « dit » l'image n'est pas soluble en soi, puisque a contrario, si la photographie peut être un art et que l'esthétique iconographique fait peser sur le chercheur un certain nombre de soupçons, elle peut aussi paradoxalement couper court aux risques d'esthétisation démesurée, de lyrisme, et donc de normativité. L'usage de la photographie peut même apparaître d'autant plus justifié dans le cas d'objets susceptibles d'être décrits dans une prose esthétisante, tels que les ambiances, les pratiques et les œuvres artistiques. C'est ainsi que certaines recherches en sociologie visuelle plaident aujourd'hui pour un usage esthétique de la photographie, à partir d'une mise sur le même plan de ces deux modes d'expression que sont le texte et l'image : « le soin particulier apporté au cadrage et au positionnement du sujet photographié par rapport à la lumière naturelle ou artificielle, le choix de l'objectif photographique, le temps de pose représentent des moyens d'expression. Lorsqu'on élabore un texte, on choisit également d'adopter une mise en forme plus ou moins sophistiquée pour exprimer une idée " (Conord, 2007, 14). Plus largement, photographier peut se justifier dans une démarche ethnographique lorsque le talent d'écriture fait défaut et empêche de restituer une description : "c'est dans les blancs de la parole [que la photographie] a sa plus grande pertinence» (Laplantine, 2007, 51). En ce sens, la photo peut être une "forme d'expérience et un mode de connaissance non verbal dans lequel il y a bien de la pensée, mais de la pensée qui n'est pas organisée de manière discursive" (Ibidem). Cette difficulté rencontrée dans l'écriture ethnographique semble par ailleurs fort justifiée lorsqu'il s'agit de restituer des ambiances et des atmosphères, ou encore le ressenti subjectif de l'observateur face à ce que " dégagent » certains enquêtés : comment (d)écrire l'impression de charisme, les traits avenants ou fermés d'un enquêté, la présence en filigrane mais déterminante d'un autre? Et «comment dire la plus grande intensité du plaisir, les minuscules inflexions des mouvements du corps et des expressions du visage pris dans ce que Raymond Depardon appelle les "temps faibles" de la vie quotidienne (Depardon 1993) ?» (Ibidem). Ici, réaliser un instantané de ce que l'on voit aide sans doute le lecteur à se représenter la scène sociale et les individus à partir desquels l'observateur aura constitué son matériau: le travail photographie devient bien un rempart à l'approximation ou à l'esthétisation. Aujourd'hui, la vraie question n'est donc plus tant celle du degré d'esthétisation des scènes relatées, que celle du sentiment d'ajustement entre une image et mon idée. Dit autrement, ce type de questionnement pourrait être aisément résolu, mais le fait qu'il ait constitué un dilemme durant la thèse est significatif de l'absence de formation, voire de l'impensé, que constitue l'utilisation possible de la photographie dans une enquête de terrain et dans l'arsenal des outils à disposition du chercheur. 


\section{Conclusion}

S. Maresca rappelle combien certains "grands noms des sciences sociales [parmi lesquels P. Bourdieu, C. Levi-Strauss, G. Tillion] vont valoriser sur le tard leur production photographique à travers la publication d'albums qui restituent des clichés souvent pris au début de leur carrière et qu'eux-mêmes n'avaient pas jugé bon d'exploiter plus avant " (Maresca, 2007, 62). Si la sociologie visuelle ou les visual studies se sont développées et codifiées ces dernières décennies, elles ne se sont pas pour autant ritualisées et imposées comme légitimes dans les méthodes d'enquêtes en sciences sociales, et en particulier en sociologie ou science politique. L'aperçu des usages "sauvages", peu rationalisés ou des non-usages, de l'image au cours de mon travail de thèse en est une illustration parmi tant d'autres. Or, l'un des effets majeurs de l'effacement des données visuelles dans les enquêtes est sans doute de passer à côté d'un potentiel méthodologique et réflexif important, à l'instar de la mise en retrait de la subjectivité du chercheur dans de nombreux travaux en sciences sociales. On peut de ce point de vue établir un parallèle entre la disparition des images des travaux finaux, et le "basculement du "je" initial, subjectif et tâtonnant [pendant l'enquête], au "on" final, expression impersonnelle d'un surmoi intellectuel assuré » (Maresca, 2000). Pourtant, avec ces « nouveau matériaux, moins orthodoxes, souvent encore difficiles à légitimer", le chercheur se trouve "confronté à de nouvelles questions méthodologiques et épistémologiques " (De Verdalle et Israël, 2002, 9). C'est dans cette optique que j'ai restitué trois types de pratiques de l'image, gagnant graduellement en réflexivité : un usage basique et banalisé (l'image carnet de terrain et l'illustration de la recherche), l'intérêt pour l'image en tant que pratique sociale, puis une étape réflexive sur l'esthétique visuelle et ses implications épistémologiques. Certes, la "nouveauté » des questionnements méthodologiques ou épistémologiques autour de l'iconographie n'est pas toujours à l'œuvre, au sens où ils recoupent dans bien des cas les logiques à l'œuvre autour d'autres dispositifs ou matériaux non-visuels. Pourtant, j'ai voulu montrer ici que quel que soit le degré d'expertise du chercheur dans le champ de la sociologie visuelle, questionner ces matériaux ne peut que lui offrir des pistes d'analyses et outils méthodologiques supplémentaires. Le cheminement individuel présenté ici, pendant la thèse et après, a en effet permis de mettre en évidence plusieurs potentiels inexploités de ces pratiques: inventaire du «vu» et de "l'invisibilisé ", production de réactions et représentations profanes sur l'objet, production de nouvelles données empiriques, autoanalyse du rapport - esthétisé, enchanté, ou désintéressé, partiel, etc. - à l'objet. Cet article n'est donc pas un plaidoyer pour un usage à tout prix des images, mais plutôt une invitation à explorer les possibles que recèle un usage « sauvage » des images. 


\section{BIBLIOGRAPHIE}

Bajard F. (2013), « Enquêter en milieu familier. Comment jouer du rapport de filiation avec le terrain ? ", Genèses, Sciences sociales et histoire, $\mathrm{n}^{\circ}$ 90, p. 7-24.

Bajard F. (2014), « L'invention de la céramique d'art. Contribution à la sociologie de la construction des groupes professionnels », Sociologie du travail, vol. 57, n 3, p. 299-321.

Bajard F. et Perrenoud M. (2013), « “Ça n'a pas de prix”. Diversité des modes de rétribution du travail des artisans d'art ", Sociétés contemporaines, vol. 91, n 3, p. 93-116.

Beaud S. et Weber F. (2010), Guide de l'enquête de terrain : produire et analyser des données ethnographiques, Paris, La Découverte.

Becker H. (2007), « Les photographies disent-elles la vérité ? », Ethnologie française, vol. 37, n 1, p. 33-42.

Bidet A. (2011), L'engagement dans le travail : qu'est-ce que le vrai boulot ?, Paris, PUF.

Bourdieu P. (1993a), « Comprendre », in Bourdieu P. (dir.), La misère du monde, Paris, Seuil, p. $1389-1447$.

Bourdieu P. (1993b), «Intérêt et désintéressement », Cours du Collège de France à la Faculté d'Anthropologie et de Sociologie de l'Université Lumière Lyon 2, les 1er et 8 décembre 1988, Cahiers de recherche du GRS.

Chauvin P.-M. et Reix F. (2015), « Sociologies visuelles. Histoire et pistes de recherche », L'Année sociologique, vol. $65, \mathrm{n}^{\circ} 1$, p. 15-41.

Collier J. (1986), Visual Anthropology: Photography as a Research Method, UNM Press.

Conord S. (2007), « Usages et fonctions de la photographie », Ethnologie française, vol. 37, n 1, p. $11-22$.

Deze A. (2013), « Pour une iconographie de la contestation », Cultures \& Conflits, vol. 3, n 91-92, p. 13-29.

Heinich N. et Shapiro R. (2012), De l'artification enquêtes sur le passage à l'art, Paris, EHESS.

Hughes E.C. (1996), Le regard sociologique : essais choisis, Paris, EHESS.

Laplantine F. (2007), «Penser en images », Ethnologie française, vol. 37, nº 1, p. 47-56.

Maresca S. (2000), « Introduction à Questions d'optique ( $n^{\circ}$ spécial du Journal des anthropologues) », Journal des anthropologues, p. 9-20.

Maresca S. (2007), « Photographes et ethnologues », Ethnologie française, vol. 37, n 1, p. 61-67.

Maresca S. (2011), « Spécimens ou individus ? », L’Homme, n 198-199, p. 67-87.

Maresca S. et Meyer M. (2013), Précis de photographie à l'usage des sociologues, Rennes, PUR.

Mauss M. (1967), Manuel d'ethnographie, Paris, Ed. sociales.

Meyer M. (2014), « Le travail des regards. Photographier les interactions et interagir avec les photographies », dans Perrenoud M. (dir.), Les mondes pluriels de Howard S. Becker, La Découverte, p. 147-166.

Papinot C. (2007), « Le "malentendu productif”. Réflexion sur la photographie comme support d'entretien », Ethnologie française, vol. 37, n 1, p. 79-86. 
Piette A. (2007), « Fondements épistémologiques de la photographie », Ethnologie française, vol. 37, $\mathrm{n}^{\circ} 1$, p. 23-28.

Pina C. et Savarese É. (à paraitre), « Pour une analyse iconographique du politique. Regards interdisciplinaires, enjeux et perspectives », in Iconographie et science politique, Paris, L'Harmattan.

Schwartz O. (1993), «L'empirisme irréductible. La fin de l'empirisme? », in Anderson N. (dir.), Le Hobo, sociologie du sans-abri, Paris, Nathan, p. 265-308.

Terrenoire J.-P. (1985), « Images et sciences sociales : l'objet et l'outil », Revue française de sociologie, $\mathrm{n}^{\circ} 26$, p. 509-527.

Verdaille L. (de) et Israël L. (2010), «Image(s) des sciences sociales (avant-propos) », Terrains \& travaux, $\mathrm{n}^{\circ} 3$, p. 7-13.

Weber M. (1965), « Essai sur le sens de la "neutralité axiologique” dans les sciences sociologiques et économiques ", Essais sur la théorie de la science.

\section{NOTES}

1. Si les réflexions sur la pratique de l'image en sciences sociales sont essentiellement réalisées à partir de terrains occidentaux (Conord, 2007, 12) la routinisation de longue date de l'usage des images concerne surtout des terrains dits " exotiques", étudiés en anthropologie, y compris de manière coloniale ou purement illustrative.

2. Ces usages "non contrôlés" des images dans les sciences sociales ont d'ailleurs sans doute "participé à délégitimer ce type de données aux yeux des sociologues » (Chauvin et Rex, 2007, 34).

3. S. Maresca identifie quatre grandes thématiques dans la bibliographie existante: «historiographique (la photographie comme trace historique); - méthodologique (la photographie comme outil de recherche); - épistémologique (la photographie comme moteur intellectuel) ; - anthropologique (la photographie comme enjeu culturel et politique) » (Maresca, 2000).

4. Il n'existe pas de recensement officiel des céramistes d'art, puisqu'ils exercent sous des statuts légaux variés. La liste la plus exhaustive reste à ce jour l' " annuaire » édité tous les quatre ans par les éditions de La revue de la céramique et du verre.

5. Les données visuelles se prêtent aussi au comptage et au traitement statistique (Chauvin et Reix, 2015, 26).

6. Site web : http://www.jeremielogeay.fr/photographies/potiers

7. M. Mauss notait, à propos de l'étude des techniques du corps et artisanales, des lieux ou encore des systèmes d'élevage : "On ne fera jamais trop de photos, à condition qu'elles soient toutes commentées et exactement situées : heure, place, distance » (Mauss, 1967, 14).

8. Voir à ce sujet (Chauvin et Reix, 2015) : les auteurs mentionnent par exemple, dans le cas du travail et des portraits de travailleurs, les travaux des photographes Bruno Fert et Eugène Atget.

9. Types « dont les descriptions littéraires ne peuvent parvenir à rendre compte de façon aussi précise et « incarnée » » (Chauvin et Reix, 2015, 31)

10. Ces portraits d'individus singuliers avaient ainsi une fonction typologique et analytique. Ils ne consistaient pas en une accumulation de spécimens assimilables «ni plus ni moins, à des planches d'herbiers ou des séries de papillons » (Maresca, 2011).

11. J'emploierai le masculin général, même si le métier s'est largement féminisé depuis les années 1990. 
12. Notons aussi que j'appliquais ce questionnement à l'expression verbale : je me suis intéressée à ce que les enquêtés donnaient à entendre, notamment dans leurs relations avec la clientèle.

13. Sur cet aspect, voir l'analyse des diverses formes de rétributions, mais aussi de valorisation, du travail des artisans d'art, notamment à partir des outils de présentation de soi, dans (Bajard et Perrenoud, 2013).

14. De même, j'aurais tout à fait pu utiliser la photographie comme support d'entretien pour questionner ce que les enquêtés qualifiaient de «beau » ou de «bon » travail, et donc renseigner les critères de professionnalité et de hiérarchisation au sein du métier.

15. En général trouvés sur les sites personnels de céramistes ou de galeries.

16. F. Laplantine propose quelques pistes de réflexion sur la relation entre écriture et photographie lorsqu'il s'agit de se mémoriser, décrire et retranscrire ce qui est vu, regardé, perçu, caché ou ressenti (Laplantine, 2007).

\section{RÉSUMÉS}

Certains sociologues - dont je fais partie - ne font pas ou peu usage de l'image, et ce (non) choix relève souvent d'un impensé. Les images sont pourtant riches d'enseignements sur le plan analytique et méthodologique, en tant que matériaux empirique, dispositifs de recherche ou support de restitution des résultats. Or, ce potentiel heuristique peut aussi valoir dans le cas d'usages «sauvages" des images, c'est-à-dire parcellaires, plus ou moins rationalisés et contrôlés, par une sociologue peu habituée à la pratique de la sociologie visuelle. Dans l'écheveau de relations sociales impliquant le chercheur, les enquêtés et les récepteurs de ces travaux de recherche, les images ont ici pu être utiles à plusieurs égards : réaliser un inventaire $d u$ « vu » et de «l'invisibilisé » par l'ethnographie, produire des discours profanes sur l'objet et donc de nouveaux matériaux empiriques, ou encore servir de support à l'analyse du rapport - esthétisé, enchanté, partiel, etc. - du chercheur à son terrain. Cet article n'est donc pas un plaidoyer pour un usage à tout prix des images, mais plutôt une invitation à explorer les possibles qu'elles recèlent y compris par des profanes de la sociologie visuelle.

Some sociologists - of which I am part - do not use (or use very few) images in their research, and such a (non) choice is often overlooked. Yet, images may provide valuable lessons in terms of methods and analysis if one considers them as empirical datas and research tools as well as materials for feeding back the results of the research. This article shows that this heuristic potential also concerns "wild uses" of images, that is, partial, more or less rationalized and controlled uses carried out by a sociologist who was not familiar of visual studies. Here, images were entangled in social relationships involving the researcher, respondents and readers/ listeners of the research, and were useful in several ways: taking an inventory of the "seen" and "unseen" within the ethnographic work, producing lay discourses about the object and therefore, new empirical datas, and support further thoughts on the - aestheticized, enchanted, partial, etc. - relation of the researcher towards its fieldwork. Thus, this article does not advocate for a use at all costs of images, but rather invites to take advantage of their possibilities included by nonexperts of visual sociology. 
INDEX

Keywords : wild uses, reflexivity, image, photography, ethnography, aestheticism, visual sociology

Mots-clés : usages « sauvages », réflexivité, image, photographie, ethnographie, esthétique, sociologie visuelle

\section{AUTEUR}

\section{FLORA BAJARD}

Flora Bajard est sociologue, chargée de recherche au CNRS au LEST (Aix Marseille Univ, CNRS, LEST, Laboratoire d'Economie et de Sociologie du Travail, Aix-en-Provence, France). Sa thèse réalisée à l'université de Lausanne (dir. Marc Perrenoud) portait sur le métier de céramiste d'art en France, à la croisée de la sociologie de l'art, de la sociologie du travail et des professions, et de la sociologie politique. Son projet de recherche actuel porte sur les expériences collectives de travail indépendant. 